\title{
Robust Finite-Time H-Infinity Control with Transients for Dynamic Positioning Ship Subject to Input Delay
}

\author{
Xiaogong Lin, Kun Liang $(\mathbb{D}$, Heng Li, Yuzhao Jiao $\mathbb{D}$, and Jun Nie \\ College of Automation, Harbin Engineering University, Harbin, China \\ Correspondence should be addressed to Kun Liang; drliangkun@126.com
}

Received 1 January 2018; Revised 4 April 2018; Accepted 16 May 2018; Published 26 June 2018

Academic Editor: Sabri Arik

Copyright (c) 2018 Xiaogong Lin et al. This is an open access article distributed under the Creative Commons Attribution License, which permits unrestricted use, distribution, and reproduction in any medium, provided the original work is properly cited.

\begin{abstract}
This paper presents the problem of robust finite-time $H \infty$ control with transients for ocean surface vessels equipped with dynamic positioning (DP) system in presence of input delay. The main objective of this work is to design a finite-time $H \infty$ state feedback controller, which ensures that all states of ship do not exceed a given threshold over a fixed time interval, with better robustness and transient performance subject to time-varying disturbance. Based on a novel augmented Lyapunov-Krasovskii-like function (LKLF) with triple integral terms and a method combining the Wirtinger inequality and reciprocally convex approach, a less conservative result is derived. In particular, an $H \infty$ performance index with nonzero initial condition is introduced to attenuate the overconservatism caused by the assumption of zero initial condition and enhance the transient performance of ship subject to external disturbance. More precisely, the controller gain matrix for the DP system can be achieved by solving the linear matrix inequalities (LMIs), which can be easily facilitated by using some standard numerical packages. Finally, a numerical simulation for a ship is proposed to verify the effectiveness and less conservatism of the controller we designed.
\end{abstract}

\section{Introductions}

With the increasing development in the ocean exploitation, dynamic positioning (DP) systems, regulating the horizontal position and heading of the vessel exclusively by means of active thrusters, have been developed for various marine and offshore applications such as drilling, salvage, pipelaying, and oil production [1]. To achieve expected trajectory tracking or positioning, various control strategies have been proposed, including robust adaptive control [2], sliding mode control [3], prescribed performance control [4], hybrid control [5], and neural network control [6]. However, in some applications, it is significant to maintain the vessel's states under some bounds, particularly of which transient performance is emphasized, during a specific time interval, for example, when facing the matter of saturations or when the task of trajectory tracking should be fulfilled in a prescribed time interval. In [7], the proposed controller can maintain the bound of ship over an infinity time interval regardless of disturbance. However, it is inconvenient to analyze and to enhance the transient performance when the operation should be arrived in short time, such as rescuing works. On the other hand, time delay is encountered in many dynamical systems and often leads to performance deterioration which engenders strongly growing interest in this topic in recent decades. In the DP system, the main kind of time delay is encountered in actuators [8], while another obvious kind of delay is the one produced between the sensors and the activation of the control mechanism [9]. The effect caused by time delay will be more significant in finite-time interval and it is the first motivation of this paper.

The classical $H \infty$ control theory [10] defines the control law, under the assumption of zero initial condition, providing the minimal value to the performance measure that is the worst-case norm of the regulated output over all exogenous signals and is applied to DP system successfully [11]. However, there exist some situations, when the initial state of ship is possibly nonzero, such as when saturation is activated or controller is switched to another one. It will cause an additional unknown disturbance [12] and the promising robustness is achieved at the expense of degraded nominal performance [13]. In [14], researchers introduced a performance measure that is the induced norm of the regulated output over all exogenous signals and initial states for finite 
and infinite horizons. Unfortunately, to the best the authors' knowledge, it has not been extended to the system with input delay. On another research frontier, various approaches, in the framework of finite-time boundedness for time-delay systems, have been developed to obtain the results with less conservatism $[15,16]$, usually indicated by the bound of state [17]. For DP system, engineers are willing to obtain control strategies which maintain the state of ship varying in a small region around the desired set-point or tracking path instead of the acceptable minimum state bound. Thus, the second motivate is to obtain a result which reduces the overconservatism caused by the assumption of zero initial condition and the loss information in the proof for time delay systems in a practical view.

Based on the discussion aforementioned, the problem of robust finite-time $H \infty$ control for DP system with input delay is studied. The main contribution of this paper lies in three aspects: Firstly, a finite-time $H \infty$ controller with transients is designed for DP systems with input delay, by solving a couple of LMIs, which can guarantee the state of ship within a desired value over a fixed time interval in the presence of time-varying disturbances. Secondly, the concept of $H \infty$ control under nonzero initial condition is introduced to time-delay system and its advantage on enhancing the transient performance will be shown in a numerical simulation compared to the $H \infty$ with zero initial condition. In particular, the results are established in forms of LMI, which can be easily facilitated by using some standard numerical packages. Thirdly, a novel augmented LKLF with triple integral terms, which contains more information is constructed; meanwhile a method combining the Wirtinger inequality and reciprocally convex approach is applied to obtain a tight bound of the integral terms of quadratic functions which may lead to a less conservative result compared to previous works. Moreover, the practical significance of this method in engineering will be demonstrated later. Finally, a numerical simulation for a ship is proposed to verify the effectiveness and advantage of the controller we designed.

The rest of this paper is organized as follows: In Section 2, the problem formulation of finite-time $H \infty$ control is detailed for vessels while some definitions and lemmas are introduced as preparation. In Section 3, the method to design finite-time $H \infty$ controller is proposed, and the proposed control schemes are simulated in Section 4. In Section 5, the conclusion is drawn.

Notation. Throughout this paper, $\mathfrak{R}^{n}$ is the $n$-dimensional Euclidean vector space, and $\mathfrak{R}^{m \times n}$ denotes the set of all $m \times n$ real matrices. For symmetric matrices $X$ and $Y$, $X>Y$ (respectively, $X \geq Y$ ) means that $X-Y$ is positive definite (respectively, positive semidefinite). The superscript " $T$ " represents the transpose. The symmetric terms in a symmetric matrix are denoted by “*”. Moreover, we use $\lambda_{\max }(\cdot)\left(\lambda_{\min }(\cdot)\right)$ to denote the maximum (minimum) eigenvalue of a symmetric matrix.

\section{Problem Formulation}

2.1. Model of DP System. At first, DP system model with three-DOP under low speed can be described as [18]

$$
\begin{aligned}
\dot{\eta}(t) & =J(\psi) v(t) \\
M \dot{v}(t)+D v(t) & =u(t-h(t))+\omega(t)
\end{aligned}
$$

where $v=\left(\begin{array}{lll}\mu & v & r\end{array}\right)$ is a vector of velocities given in the body-fixed coordinate system and $\eta=\left(\begin{array}{lll}X & Y & \psi\end{array}\right)$ is the position and orientation of the vessel with respect to an inertial reference coordinate system. $u(t)$ is a control vector of forces and moments provided by the propulsion systems. $h(t)$ is a time-varying function that expresses the actuator delay and satisfies $h(t)<h \dot{h}(t)<\mu . \omega(t)$ is the disturbance input of the system and satisfies the condition of $\int_{0}^{\infty} \omega(t)^{T} \omega(t) d t<$ d. $J(\psi)$ is the transformation matrix between the inertial and body-fixed coordinate frames. The inertia matrix $M$ includes hydrodynamic added inertia, and $D$ is a strictly positive damping matrix due to linear wave drift damping and laminar friction. Then, the structures of the matrices $J(\psi), M$, and $D$ can be explicitly given as follows:

$$
\begin{aligned}
J(\psi) & =\left(\begin{array}{ccc}
\cos (\psi) & -\sin (\psi) & 0 \\
\sin (\psi) & \cos (\psi) & 0 \\
0 & 0 & 1
\end{array}\right) \\
M & =\left(\begin{array}{ccc}
m-X_{\dot{u}} & 0 & 0 \\
0 & m-Y_{\dot{v}} & m x_{g}-Y_{\dot{r}} \\
0 & m x_{g}-Y_{\dot{r}} & I_{z}-N_{\dot{r}}
\end{array}\right) \\
D & =-\left(\begin{array}{ccc}
X_{u} & 0 & 0 \\
0 & Y_{v} & Y_{r} \\
0 & N_{v} & N_{r}
\end{array}\right)
\end{aligned}
$$

where $X_{u}, Y_{v}, Y_{r}, N_{r}, X_{\dot{u}}, Y_{\dot{v}}, Y_{\dot{r}}$, and $N_{\dot{r}}$ are the hydrodynamic parameters of the vessel. $m$ is the mass of the ship, and $I_{z}$ is the moment of inertia about the yaw rotation. $x_{g}$ is the vertical distance from coordinate origin to center of gravity in body-fixed frame.

To simplify the model, some assumptions are introduced first.

Assumption 1. All the parameters of state are available.

Assumption 2. The roll and pitch angles are small enough; for DP system, it is a reasonable assumption.

Under assumption 2, we can obtain the simplified equation in form of state-space [19]

$$
\begin{aligned}
& \dot{x}(t)=A x(t)+B_{1} u(t-h(t))+B_{2} \omega(t) \\
& z(t)=C x(t)+D u(t-h(t))
\end{aligned}
$$

where

$$
\begin{aligned}
x(t) & =(\eta(t) \\
A & =\left(\begin{array}{cc}
0 & I \\
0 & -M^{-1} D
\end{array}\right)
\end{aligned}
$$




$$
B_{1}=B_{2}=\left(\begin{array}{c}
0 \\
M^{-1}
\end{array}\right) \text {. }
$$

Under assumption 1, we design the full-state feedback as

$$
u(t-h(t))=K x(t-h(t))
$$

so the (1) and (2) can be rewritten as

$$
\begin{aligned}
& \dot{x}(t)=A x(t)+B_{1} K x(t-h(t))+B_{2} \omega(t) \\
& z(t)=C x(t)+D K x(t-h(t)) .
\end{aligned}
$$

Remark 3. In the situation of DP motion, the motion in heave, roll, and pitch will be ignored since we focus on the motion on the surface of sea. Meanwhile, velocity of vessel is low enough, so the Coriolis-Centripetal matrix and nonlinearities in damping matrix can be neglected [18].

Remark 4. In measurement subsystem of DP system, various sensors are installed to obtain the state of the vessel motion accurately, including global position system for the vessel' $s$ position, gyrocompass for the vessel' s heading, and attitude sensor for the pitch and roll. Meanwhile, data fusion technology is applied to DP system to obtain more accurate state information of vessel motion [18].

2.2. Preliminaries. In the sequel, some definitions and lemmas are introduced to obtain the results.

Definition 5 ((FTB), see [20]). Given a positive definite matrix $R$ and three positive constants $c_{1}, c_{2}, T$ with $c_{1}<$ $c_{2}$, the time-delay system (4) with $u(t)=0$ is said to be finite-time boundedness with respect to $\left(\begin{array}{llllll}c_{1} & c_{2} & d & T & \tau & R\end{array}\right)$, if $\sup _{-\tau<\theta<0}\left\{x^{T}(\theta) R x(\theta), \dot{x}^{T}(\theta) R \dot{x}(\theta)\right\}<c_{1} \Rightarrow x^{T}(t) R x(t)<$ $c_{2}, \forall t \in[0, T], \forall \omega(t): \int_{0}^{T} \omega(t)^{T} \omega(t) d t<d$.

Definition 6. Given $\gamma>0$, time-delay systems (4) and (5) are said to be $H \infty$ - FTB under nonzero initial condition $(H \infty$ FTB) with ( $\left(\begin{array}{lllllll}c_{1} & c_{2} & d & \gamma & T & \tau & R\end{array}\right)$, if the following conditions are satisfied:

(1) time-delay system (4) with $u(t)=0$ is FTB;

(2) under the initial condition that $x(0) \neq 0$, output $z(t)$ satisfies

$$
\begin{aligned}
& \Gamma \\
& =\sup _{\|\omega(t)\|_{2,[0, T]^{2}}{ }^{+}+x(0)^{T} S x(0) \neq 0}\left[\frac{\|z(t)\|_{2,[0, T]}{ }^{2}}{\|\omega(t)\|_{2,[0, T]^{2}}+x(0)^{T} S x(0)}\right]^{1 / 2} \\
& <\gamma
\end{aligned}
$$

where $\gamma$ is a prescribed scalar and $S$ is a weighting diagonal matrix that penalizes the effect caused by the initial state.

Remark 7. It is necessary to distinguish between finite-time stability and finite-time attractiveness [21]. The first concept is to maintain system states within a given boundary in a specified time interval $[22,23]$, while the latter describes the fact that system state reaches the equilibrium point of system in a finite time [24].

Remark 8. Similar to [13], this definition of $H \infty$ performance, which depends on the initial condition, has been extended to linear system with acceptable maximal delay bound. The performance measure is parametrised by a weighting matrix $S$ reflecting the relative importance of the uncertainty in the initial state contrary to the uncertainty in the exogenous disturbance. When $S=0$, it will reduce into a sort of $H \infty$ control with zero initial condition [25-27].

Lemma 9 (see [28]). For any matrix $P>0$ and a differentiable signal $x$ in $[-a,-b] \rightarrow \mathfrak{R}^{n}$, the following inequality holds:

$$
\begin{aligned}
& \int_{-a}^{-b} \dot{x}^{T}(u) S_{1} \dot{x}(u) d s \geq \frac{1}{a-b} \\
& \cdot \Pi_{1}\left(S_{1}, x^{T}(-b), x^{T}(-a), \frac{1}{a-b} \int_{-a}^{-b} x^{T}(u) d u\right)
\end{aligned}
$$

where

$$
\begin{aligned}
& \Pi_{1}\left(S_{1}, \alpha, \beta, \gamma\right) \\
& \quad=\left(\begin{array}{c}
(\alpha-\beta)^{T} \\
(\alpha+\beta-2 \gamma)^{T}
\end{array}\right)^{T}\left(\begin{array}{cc}
S_{1} & 0 \\
* & 3 S_{1}
\end{array}\right)\left(\begin{array}{c}
(\alpha-\beta)^{T} \\
(\alpha+\beta-2 \gamma)^{T}
\end{array}\right) .
\end{aligned}
$$

Lemma 10 (see [29]). For constant matrices $S_{1}>0, S_{2}>0$ and constant scalars $a>b$, the following inequalities hold for all continuously function $x$ in $[-a,-b] \rightarrow \mathfrak{R}^{n}$ :

$$
\begin{aligned}
& \int_{-a}^{-b} \int_{t+\theta}^{t-b} \dot{x}^{T}(u) S_{2} \dot{x}(u) d u d \theta \\
& \quad \geq \Pi_{2}\left(S_{2}, x^{T}(t-b), \frac{1}{a-b} \int_{t-a}^{t-b} x^{T}(u) d u, \frac{1}{(a-b)^{2}}\right. \\
& \left.\quad \cdot \int_{-a}^{-b} \int_{t+\theta}^{t-b} x^{T}(u) d u d \theta\right) \\
& \int_{-a}^{-b} \int_{t-a}^{t+\theta} \dot{x}^{T}(u) S_{3} \dot{x}(u) d u d \theta \\
& \quad \geq \Pi_{3}\left(S_{3}, x^{T}(t-a), \frac{1}{a-b} \int_{t-a}^{t-b} x^{T}(u) d u, \frac{1}{(a-b)^{2}}\right. \\
& \left.\cdot \int_{-a}^{-b} \int_{t+\theta}^{t-b} x^{T}(u) d u d \theta\right)
\end{aligned}
$$

where

$$
\begin{array}{r}
\Pi_{2}\left(S_{2}, \alpha, \beta, \gamma\right)=\left(\begin{array}{c}
(\alpha-\beta)^{T} \\
\left(\frac{\alpha}{2}-\gamma\right)^{T}
\end{array}\right)^{T} \\
\cdot\left(\begin{array}{cc}
6 S_{2} & -12 S_{2} \\
* & 36 S_{2}
\end{array}\right)\left(\begin{array}{c}
(\alpha-\beta)^{T} \\
\left(\frac{\alpha}{2}-\gamma\right)^{T}
\end{array}\right)
\end{array}
$$




$$
\begin{array}{r}
\Pi_{3}\left(S_{3}, \alpha, \beta, \gamma\right)=\left(\begin{array}{c}
(\alpha-\beta)^{T} \\
\left(\frac{\alpha}{2}-\beta+\gamma\right)^{T}
\end{array}\right)^{T} \\
\cdot\left(\begin{array}{cc}
6 S_{3} & -12 S_{3} \\
* & 36 S_{3}
\end{array}\right)\left(\begin{array}{c}
(\alpha-\beta)^{T} \\
\left(\frac{\alpha}{2}-\beta+\gamma\right)^{T}
\end{array}\right) .
\end{array}
$$

Lemma 11 (see [30]). Let $f_{1}, f_{2}, \ldots, f_{N}: \mathfrak{R}^{m} \mapsto \mathfrak{R}$ have positive values in an open subset $D$ of $\Re^{m}$. Then the reciprocally convex combination of $f_{i}$ over $D$ satisfies

$$
\begin{aligned}
\min _{\left\{\alpha_{i} \mid \alpha_{i}>0, \sum_{i} \alpha_{i}=1\right\}} & \sum_{i} \frac{1}{\alpha_{i}} f_{i}(t)=\sum_{i} f_{i}(t)+\max _{g_{i, j}(t)} \sum_{i \neq j} g_{i, j}(t) \\
& \text { subject to }\left\{g_{i, j}: R^{m} \longmapsto R, g_{j, i}(t)=g_{i, j}(t),\left[\begin{array}{cc}
f_{i}(t) & g_{i, j}(t) \\
g_{j, i}(t) & f_{j}(t)
\end{array}\right] \geq 0\right\} .
\end{aligned}
$$

Lemma 12 (see [31]). For any matrix $S_{4}>0$ and a vector function $x$ in $[-a,-b] \rightarrow \mathfrak{R}^{n}$, if the integrals concerned are well defined, then the following inequality holds:

$$
\begin{aligned}
& \int_{-a}^{-b} x^{T}(s) S_{4} x(s) d s \\
& \quad \geq \frac{1}{a-b}\left(\int_{-a}^{-b} x^{T}(s) d s\right) S_{4}\left(\int_{-a}^{-b} x(s) d s\right) .
\end{aligned}
$$

2.3. Control Objective. Finally, the objective of this paper is to derive the control gain $K$ such that

(1) the closed-loop system (8) with $u(t)=0$ is FTB;

(2) under given nonzero initial condition, the closed system (8) and (9) guarantees that $J=\int_{0}^{T} z^{T}(s) z(s)-$ $\gamma^{2} \omega^{T}(s) \omega(s)-\gamma^{2}(1 / T) x^{T}(0) S x(0) d s<0$ for all nonzero $\omega(t)$ and $t \in[0, T]$.

\section{Main Results}

The designing of the robust finite-time Ho controller for the DP system with input delay is divided into three steps.

3.1. FTB Analysis of DP System. Firstly, the result guaranteeing the FTB of DP system is established in this subsection.

Theorem 13. Given five positive scalars $\alpha, \beta, c_{1}, c_{2}, T$ and positive define matrix $R$, the finite-time boundedness problem of system (4) is solvable if there exist positive scalars $\theta_{l}(l=$ $1,2, \cdots, 8)$ and matrices $P_{11}>0, P_{22}>0, W>0, Q_{i}>$ $0(i=1,2), U_{j}>0(j=1,2), M>0, Z_{1}, Z_{2}$ with appropriate dimensions, satisfying the following conditions:

$$
\begin{gathered}
\Omega=\left(\Omega_{i j}\right)_{8 \times 8}<0 \\
\left(\begin{array}{ll}
W & Z_{1} \\
* & W
\end{array}\right)>0
\end{gathered}
$$

$$
\begin{aligned}
& \left(\begin{array}{cc}
3 W & Z_{2} \\
* & 3 W
\end{array}\right)>0 \\
& c_{1} \theta_{2}+h^{2} c_{1} \theta_{3}+\rho h c_{1} \theta_{4}+\rho h c_{1} \theta_{5}+\frac{1}{2} \rho h^{3} c_{1} \theta_{6} \\
& \quad+\frac{1}{6} \rho h^{3} c_{1} \theta_{7}+\frac{1}{3} \rho h^{3} c_{1} \theta_{8}+\beta \theta_{9} d<e^{-\alpha T} c_{2} \theta_{1}
\end{aligned}
$$

where

$$
\begin{aligned}
\Omega_{11}= & A^{T} P_{11}+P_{11} A-\alpha P_{11}+Q_{1}+Q_{2} \\
& +A^{T} \frac{1}{2} h^{2}\left(U_{1}+U_{2}+2 W\right) A-4 \rho W-3 \rho U_{1} \\
& -9 \rho U_{2}, \\
\Omega_{22}= & -\rho Q_{2}-4 \rho W-6 \rho U_{2}, \\
\Omega_{33}= & K^{T} B_{1}^{T} \frac{1}{2} h^{2}\left(2 W+U_{1}+U_{2}\right) B_{1} K-8 \rho W \\
& +\rho Z_{1}^{T}+\rho Z_{1}-\rho Z_{2}^{T}-\rho Z_{2}-(1-\mu) Q_{2}, \\
\Omega_{44}= & B_{2}^{T} \frac{1}{2} h^{2}\left(2 W+U_{1}+U_{2}\right) B_{2}-\beta M, \\
\Omega_{66}= & -12 \rho W, \\
\Omega_{77}= & -12 \rho W, \\
\Omega_{55}= & \alpha h^{2} P_{22}-18 \rho U_{2}-6 \rho U_{1}, \\
\Omega_{88}= & -36 \rho U_{1}-36 \rho U_{2}, \\
\Omega_{12}= & \rho Z_{1}-\rho Z_{2}+6 \rho U_{2}, \\
\Omega_{15}= & h P_{22}+12 \rho U_{2}, \\
\Omega_{16}= & 6 \rho W \\
\Omega_{17}= & 2 \rho Z_{2},
\end{aligned}
$$




$$
\begin{aligned}
& \Omega_{13}= P_{11} B_{1} K+A^{T} \frac{1}{2} h^{2}\left(2 W+U_{1}+U_{2}\right) B_{1} K-2 \rho W \\
&-\rho Z_{1}-\rho Z_{2}, \\
& \Omega_{14}= P_{11} B_{2}+A^{T} \frac{1}{2} h^{2}\left(2 W+U_{1}+U_{2}\right) B_{2}, \\
& \Omega_{18}=-18 \rho U_{2}+6 \rho U_{1}, \\
& \Omega_{23}=-\rho Z_{1}^{T}-\rho Z_{2}^{T}-2 \rho W, \\
& \Omega_{25}=-h P_{22}-6 \rho U_{2}, \\
& \Omega_{26}= 2 \rho Z_{2}^{T}, \\
& \Omega_{27}= 6 \rho W, \\
& \Omega_{28}= 12 \rho U_{2}, \\
& \Omega_{34}= K^{T} B_{1}{ }^{T} \frac{1}{2} h^{2}\left(2 W+U_{1}+U_{2}\right) B_{2}, \\
& \Omega_{36}= 6 \rho W+2 \rho Z_{2}^{T}, \\
& \Omega_{37}= 6 \rho W+2 \rho Z_{2}, \\
& \Omega_{58}= 12 \rho U_{1}+24 \rho U_{2}, \\
& \Omega_{67}=-4 \rho Z_{2}, \\
& \Omega_{24}= \Omega_{35}=\Omega_{38}=\Omega_{45}=\Omega_{46}=\Omega_{47}=\Omega_{48}=\Omega_{56} \\
&= \Omega_{57}=\Omega_{68}=\Omega_{78}=0, \\
& \theta_{1}= \lambda_{\min }\left(\widetilde{P}_{11}\right) \\
& \theta_{2}= \lambda_{\max }\left(\widetilde{P}_{11}\right), \\
& \theta_{3}= \lambda_{\max }\left(\widetilde{P}_{22}\right), \\
& \theta_{4}= \lambda_{\max }\left(\widetilde{Q}_{1}\right), \\
& \theta_{5}= \lambda_{\max }\left(\widetilde{Q}_{2}\right), \\
& \theta_{6}= \lambda_{\max }(\widetilde{W}), \\
&\left.\theta_{1}\right), \\
&\left.\theta_{7}\right) \\
&=
\end{aligned}
$$

$$
\begin{aligned}
\widetilde{P}_{11} & =R^{-1 / 2} P_{11} R^{-1 / 2}, \\
\widetilde{P}_{22} & =R^{-1 / 2} P_{22} R^{-1 / 2}, \\
\widetilde{Q}_{i} & =R^{-1 / 2} Q_{i} R^{-1 / 2} \quad(i=1,2,3), \\
\widetilde{W} & =R^{-1 / 2} W R^{-1 / 2}, \\
\widetilde{U}_{j} & =R^{-1 / 2} U_{j} R^{-1 / 2} \quad(j=1,2), \\
0 & <\theta_{1} I<P_{11}<\theta_{2} I, \\
0 & <P_{22}<\theta_{3} I, \\
0 & <Q_{1}<\theta_{4} I, \\
0 & <Q_{2}<\theta_{5} I, \\
0 & <W<\theta_{6} I, \\
0 & <U_{1}<\theta_{7} I, \\
0 & <U_{2}<\theta_{8} I, \\
0 & <M<\theta_{9} I .
\end{aligned}
$$

Proof. Consider the candidate augment LKLF as follows:

$$
\begin{aligned}
V(t)= & V_{1}(t)+V_{2}(t)+V_{3}(t)+V_{4}(t) \\
V_{1}(t)= & \varepsilon(t)^{T} P \varepsilon(t) \\
V_{2}(t)= & \int_{t-h}^{t} e^{\alpha(t-s)} x(s)^{T} Q_{1} x(s) d s \\
& +\int_{t-h(t)}^{t} e^{\alpha(t-s)} x(s)^{T} Q_{2} x(s) d s \\
V_{3}(t)= & h \int_{-h}^{0} \int_{t+\theta}^{t} e^{\alpha(t-s)} \dot{x}(s)^{T} W \dot{x}(s) d s d \theta \\
V_{4}(t)= & \int_{-h}^{0} \int_{\theta}^{0} \int_{t+\beta}^{t} e^{\alpha(t-s)} \dot{x}(s)^{T} U_{1} \dot{x}(s) d s d \beta d \theta \\
& +\int_{-h}^{0} \int_{-h}^{\theta} \int_{t+\beta}^{t} e^{\alpha(t-s)} \dot{x}(s)^{T} U_{2} \dot{x}(s) d s d \beta d \theta
\end{aligned}
$$

where $\varepsilon(t)=\left(x(t) \int_{t-h}^{t} x(s) d s\right) P=\left(\begin{array}{cc}P_{11} & 0 \\ * & P_{22}\end{array}\right)$.

To deal with the formulas conveniently, let us provide such definitions:

$\xi(t)$

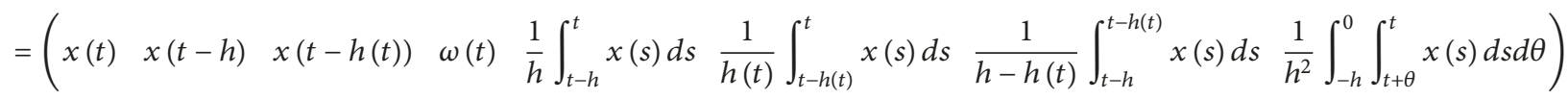

$$
\begin{aligned}
& l_{k}^{T}=\left(\begin{array}{lll}
\frac{0 \cdots 0}{k-1} & 1 & \underset{8-k}{0 \cdots 0}
\end{array}\right) \text {. }
\end{aligned}
$$


The time-derivative of $V(t)$ along system (4) can be bounded as

$$
\begin{aligned}
\dot{V}_{1}(t) & =\alpha V_{1}(t)+\dot{\varepsilon}(t)^{T} P \varepsilon(t)+\varepsilon(t)^{T} P \dot{\varepsilon}(t)-\alpha V_{1}(t) \\
\dot{V}_{2}(t) & \\
= & \alpha V_{2}(t)+x(t)^{T} Q_{1} x(t) \\
& -e^{\alpha h} x(t-h)^{T} Q_{1} x(t-h)+x(t)^{T} Q_{2} x(t) \\
& -(1-\dot{h}(t)) e^{\alpha h(t)} x(t-h(t))^{T} Q_{2} x(t-h(t)) \\
\leq & \alpha V_{2}(t)+x(t)^{T} Q_{1} x(t) \\
& -e^{\alpha h} x(t-h)^{T} Q_{1} x(t-h)+x(t)^{T} Q_{2} x(t) \\
& -(1-\mu) x(t-h(t))^{T} Q_{2} x(t-h(t))
\end{aligned}
$$$$
\dot{V}_{3}(t)
$$$$
=\alpha V_{3}(t)+h^{2} \dot{x}(t)^{T} W_{2} \dot{x}(t)
$$$$
-e^{\alpha h} h \int_{t-h}^{t} \dot{x}(s)^{T} W_{2} \dot{x}(s) d s
$$

$$
\dot{V}_{4}(t)
$$

$$
\begin{aligned}
= & \alpha V_{4}(t)+\frac{1}{2} h^{2} \dot{x}(t)^{T} U_{1} \dot{x}(t) \\
& -e^{\alpha h} \int_{-h}^{0} \int_{t+\beta}^{t} \dot{x}(s)^{T} U_{1} \dot{x}(s) d s d \beta \\
& +\frac{1}{2} h^{2} \dot{x}(t)^{T} U_{2} \dot{x}(t) \\
& -e^{\alpha h} \int_{-h}^{0} \int_{t-h}^{t+\beta} \dot{x}(s)^{T} U_{2} \dot{x}(s) d s d \beta .
\end{aligned}
$$

Invoking Lemma 9, we can obtain

$$
\begin{aligned}
\dot{V}_{3}(t)= & \alpha V_{3}(t)+h^{2} \dot{x}(t)^{T} W \dot{x}(t) \\
& -e^{\alpha h} h \int_{t-h(t)}^{t} \dot{x}(s)^{T} W \dot{x}(s) d s \\
& -e^{\alpha h} h \int_{t-h}^{t-h(t)} \dot{x}(s)^{T} W \dot{x}(s) d s \\
\leq & \alpha V_{3}(t)+h^{2} \dot{x}(t)^{T} W \dot{x}(t) \\
& -e^{\alpha h} \frac{h}{h(t)}{\omega_{1}}^{T} \Lambda_{1} \omega_{1}-e^{\alpha h} \frac{h}{h-h(t)} \omega_{2}^{T} \Lambda_{2} \omega_{2}
\end{aligned}
$$

where

$$
\begin{aligned}
& {\omega_{1}}^{T}=\xi^{T}(t)\left(\begin{array}{c}
\left(l_{1}^{T}-l_{3}^{T}\right)^{T} \\
\left(l_{1}^{T}+l_{3}^{T}-2 l_{6}^{T}\right)^{T}
\end{array}\right)^{T} \\
& \omega_{2}{ }^{T}=\xi^{T}(t)\left(\begin{array}{c}
\left(l_{3}^{T}-l_{2}^{T}\right)^{T} \\
\left(l_{3}{ }^{T}+l_{2}{ }^{T}-2 l_{7}^{T}\right)
\end{array}\right)^{T}
\end{aligned}
$$

$$
\Lambda_{1}=\Lambda_{2}=\left(\begin{array}{cc}
W & 0 \\
* & 3 W
\end{array}\right)
$$

In order to obtain a tighter bound of integral term, Lemma 11 is applied to (32) as follows:

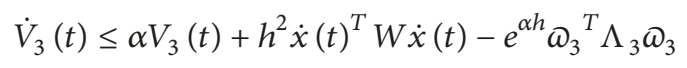

where ${\omega_{3}}^{T}=\left(\begin{array}{c}\omega_{1}{ }^{T} \\ \omega_{2}{ }^{T}\end{array}\right), \Lambda_{3}=\left(\begin{array}{cccc}W & Z_{1} & 0 & 0 \\ * & W & 0 & 0 \\ * & * & 3 W & Z_{2} \\ * & * & * & 3 W\end{array}\right)$. ity

By applying Lemma 10 to (31), we can obtain the inequal-

$$
\begin{aligned}
\dot{V}_{4}(t) \leq & \alpha V_{4}(t)+\frac{1}{2} h^{2} \dot{x}(t)^{T} U_{1} \dot{x}(t) \\
& +\frac{1}{2} h^{2} \dot{x}(t)^{T} U_{2} \dot{x}(t)-e^{\alpha h}{\omega_{4}{ }^{T} \Lambda_{4} \omega_{4}}
\end{aligned}
$$

where

$$
\begin{gathered}
\omega_{4}^{T}=\xi^{T}(t)\left(\begin{array}{c}
\left(l_{1}^{T}-l_{2}^{T}\right)^{T} \\
\left(\frac{1}{2} l_{1}^{T}-l_{8}^{T}\right)^{T} \\
\left(l_{2}^{T}-l_{5}^{T}\right)^{T} \\
\left(\frac{1}{2} l_{2}^{T}-l_{5}^{T}+l_{8}^{T}\right)^{T}
\end{array}\right) \\
\Lambda_{4}=\left(\begin{array}{cccc}
6 U_{1} & -12 U_{1} & 0 & 0 \\
* & 36 U_{1} & 0 & 0 \\
* & * & 6 U_{2} & -12 U_{2} \\
* & * & * & 36 U_{2}
\end{array}\right) .
\end{gathered}
$$

Combining (28), (29), (34), and (35) with the definition of $\rho=e^{\alpha h}$, we can obtain

$$
\dot{V}-\alpha V-\beta \omega^{T}(t) M \omega(t)<\xi^{T}(t) \Omega \xi(t) .
$$

Assuming that $\Omega<0$ and integrating the left part of inequality (37),

$$
\begin{aligned}
V(t) & <e^{\alpha t} V(0)+\beta \int_{0}^{t} e^{\alpha(t-s)} \omega^{T}(s) M \omega(s) d s \\
& <e^{\alpha T}\left(V(0)+\beta \lambda_{\max }(M) d\right) .
\end{aligned}
$$
has

Invoking the Jensen inequality shown in Lemma 12, one

$$
\begin{aligned}
V(0) \leq & x(0)^{T} P_{11} x(0)+h \int_{-h}^{0} x(s)^{T} P_{22} x(s) d s \\
& +\rho \int_{-h}^{0} x(s)^{T} Q_{1} x(s) d s \\
& +\rho \int_{-h(t)}^{0} x(s)^{T} Q_{2} x(s) d s
\end{aligned}
$$




$$
\begin{aligned}
& +h \rho \int_{-h}^{0} \int_{\theta}^{0} \dot{x}(s)^{T} W \dot{x}(s) d s d \theta \\
& +\rho \int_{-h}^{0} \int_{\theta}^{0} \int_{\beta}^{0} \dot{x}(s)^{T} U_{1} \dot{x}(s) d s d \beta d \theta \\
& +\rho \int_{-h}^{0} \int_{-h}^{\theta} \int_{\beta}^{0} \dot{x}(s)^{T} U_{2} \dot{x}(s) d s d \beta d \theta \\
& \leq \lambda_{\max }\left(\widetilde{P}_{11}\right) c_{1}+h \lambda_{\max }\left(\widetilde{P}_{22}\right) h c_{1} \\
& +\rho \lambda_{\max }\left(\widetilde{Q}_{1}\right) h c_{1}+\rho \lambda_{\max }\left(\widetilde{Q}_{2}\right) h c_{1} \\
& +\rho \lambda_{\max }(\widetilde{W}) \frac{1}{2} h^{3} c_{1}+\rho \lambda_{\max }\left(\widetilde{U}_{1}\right) \frac{1}{6} h^{3} c_{1} \\
& +\rho \lambda_{\max }\left(\widetilde{U}_{2}\right) \frac{1}{3} h^{3} c_{1}
\end{aligned}
$$

Based on inequalities (38) and (39), we have

$$
\begin{aligned}
V(t) & >x^{T}(t) P_{11} x(t)=x^{T}(t) R^{1 / 2} \widetilde{P}_{11} R^{1 / 2} x(t) \\
& >\lambda_{\min }\left(\widetilde{P}_{11}\right) x^{T}(t) R x(t) .
\end{aligned}
$$

Therefore, conditions (17) to (20) can guarantee the FTB of system (4). This completes the proof.

Remark 14. Among the existing approaches, there are two threads: one is to construct a novel LKLF that involves more information of delay; the other is to find a tighter estimation of upper bound for cross terms coming from the derivative of the LKLF. In this paper, these two techniques are applied to obtain the result. In addition, the less conservatism in practical engineering will be shown in the simulations which are always ignored in most literature sources.

3.2. Controller Design. In this subsection, we focus on the problem of finite-time $H \infty$ state feedback designing based on Theorem 13, that is, designing a state feedback controller in the form of (7) such that the resulting DP system satisfies the control objective proposed in Section 1.

Theorem 15. For given positive $\alpha, c_{1}, c_{2}, T, \gamma$ and matrices $R>$ $0, S \geq 0$, if there exist positive scalars $\theta_{l}(l=1,2, \cdots, 8)$ and matrices $\bar{P}_{11}>0, \bar{P}_{22}>0, \bar{W}>0, \bar{Q}_{i}>0(i=1,2), \bar{U}_{j}>$ $0(j=1,2), Y, \bar{Z}_{1}, \bar{Z}_{2}$ with appropriate dimensions, satisfying the following conditions:

$$
\begin{aligned}
& \widetilde{\Pi}=\left(\widetilde{\Pi}_{i j}\right)_{12 \times 12}<0 \\
& \Sigma=\left(\Sigma_{i j}\right)_{9 \times 9}<0 \\
&\left(\begin{array}{cc}
\bar{W} & \bar{Z}_{1} \\
* & \bar{W}
\end{array}\right)>0 \\
&\left(\begin{array}{cc}
3 \bar{W} & \bar{Z}_{2} \\
* & 3 \bar{W}
\end{array}\right)>0
\end{aligned}
$$

$$
\begin{aligned}
I-\gamma^{2} S \bar{P}_{11} & <0 \\
0 & <H_{1}<\bar{P}_{11}<H_{2}, \\
0 & <\bar{P}_{22}<H_{3}, \\
0 & <\bar{Q}_{1}<H_{4}, \\
0 & <\bar{Q}_{2}<H_{5} \\
0 & <\bar{W}_{6}<H_{6} \\
0 & <\bar{U}_{1}<H_{7}, \\
0 & <\bar{U}_{2}<H_{8},
\end{aligned}
$$

where

$$
\begin{aligned}
& \widetilde{\Pi}_{11}=\bar{P}_{11} A^{T}+A \bar{P}_{11}-\alpha \bar{P}_{11}+\bar{Q}_{1}+\bar{Q}_{2}-4 \rho \bar{W} \\
& -3 \rho \bar{U}_{1}-9 \rho \bar{U}_{2} \text {, } \\
& \widetilde{\Pi}_{44}=-\gamma^{2} I \text {, } \\
& \widetilde{\Pi}_{22}=-\rho \bar{Q}_{2}-4 \rho \bar{W}-6 \rho \bar{U}_{2} \text {, } \\
& \widetilde{\Pi}_{33}=-8 \rho \bar{W}+\rho \bar{Z}_{1}^{T}+\rho \bar{Z}_{1}-\rho \bar{Z}_{2}^{T}-\rho \bar{Z}_{2} \\
& -(1-\mu) \bar{Q}_{2} \text {, } \\
& \widetilde{\Pi}_{13}=B_{1} Y-2 \rho \bar{W}-\rho \bar{Z}_{1}-\rho \bar{Z}_{2} \text {, } \\
& \widetilde{\Pi}_{14}=B_{2} \text {, } \\
& \widetilde{\Pi}_{66}=-12 \rho \bar{W} \text {, } \\
& \widetilde{\Pi}_{77}=-12 \rho \bar{W}, \\
& \widetilde{\Pi}_{55}=\alpha h^{2} \bar{P}_{22}-18 \rho \bar{U}_{2}-6 \rho \bar{U}_{1}, \\
& \widetilde{\Pi}_{88}=-36 \rho \bar{U}_{1}-36 \rho \bar{U}_{2} \text {, } \\
& \widetilde{\Pi}_{12}=\rho \bar{Z}_{1}-\rho \bar{Z}_{2}+6 \rho \bar{U}_{2}, \\
& \widetilde{\Pi}_{15}=h \bar{P}_{22}+12 \rho \bar{U}_{2} \text {, } \\
& \widetilde{\Pi}_{16}=6 \rho \bar{W}, \\
& \widetilde{\Pi}_{17}=2 \rho \bar{Z}_{2} \text {, } \\
& \widetilde{\Pi}_{25}=-h \bar{P}_{22}-6 \rho \bar{U}_{2} \text {, } \\
& \widetilde{\Pi}_{18}=-18 \rho \bar{U}_{2}+6 \rho \bar{U}_{1}, \\
& \widetilde{\Pi}_{26}=2 \rho \bar{Z}_{2}^{T} \text {, } \\
& \widetilde{\Pi}_{23}=-\rho \bar{Z}_{1}^{T}-\rho \bar{Z}_{2}^{T}-2 \rho \bar{W}, \\
& \widetilde{\Pi}_{27}=6 \rho \bar{W}, \\
& \widetilde{\Pi}_{28}=12 \rho \bar{U}_{2} \text {, }
\end{aligned}
$$




$$
\begin{aligned}
& \widetilde{\Pi}_{36}=6 \rho \bar{W}+2 \rho \bar{Z}_{2}^{T} \text {, } \\
& \widetilde{\Pi}_{37}=6 \rho \bar{W}+2 \rho \bar{Z}_{2} \text {, } \\
& \widetilde{\Pi}_{58}=12 \rho \bar{U}_{1}+24 \rho \bar{U}_{2} \text {, } \\
& \widetilde{\Pi}_{67}=-4 \rho \bar{Z}_{2} \text {, } \\
& \widetilde{\Pi}_{19}=\frac{1}{2} h \bar{P}_{11} A^{T}, \\
& \widetilde{\Pi}_{39}=\frac{1}{2} h Y^{T} B_{1}{ }^{T}, \\
& \widetilde{\Pi}_{49}=\frac{1}{2} h B_{2}{ }^{T}, \\
& \widetilde{\Pi}_{1,10}=\frac{1}{2} h \bar{P}_{11} A^{T}, \\
& \widetilde{\Pi}_{3,10}=\frac{1}{2} h Y^{T} B_{1}{ }^{T}, \\
& \widetilde{\Pi}_{4,10}=\frac{1}{2} h B_{2}{ }^{T}, \\
& \widetilde{\Pi}_{1,11}=h \bar{P}_{11} A^{T} \text {, } \\
& \widetilde{\Pi}_{3,11}=h Y^{T} B_{1}{ }^{T}, \\
& \widetilde{\Pi}_{4,11}=h B_{2}{ }^{T} \text {, } \\
& \widetilde{\Pi}_{1,12}=\bar{P}_{11} C^{T} \text {, } \\
& \widetilde{\Pi}_{3,12}=Y^{T} D^{T} \text {, } \\
& \widetilde{\Pi}_{99}=\frac{1}{2} \bar{U}_{1}-\bar{P}_{11}, \\
& \widetilde{\Pi}_{10,10}=\frac{1}{2} \bar{U}_{2}-\bar{P}_{11} \text {, } \\
& \widetilde{\Pi}_{11,11}=\bar{W}-2 \bar{P}_{11} \text {, } \\
& \widetilde{\Pi}_{12,12}=-e^{-\alpha T} I,
\end{aligned}
$$

others

$$
\begin{aligned}
& \widetilde{\Pi}_{i j}=0, \\
& \Sigma_{11}=-e^{-\alpha T} c_{2} H_{1}, \\
& \Sigma_{22}=-H_{2}, \\
& \Sigma_{33}=-H_{3}, \\
& \Sigma_{44}=-H_{4}, \\
& \Sigma_{55}=-H_{5}, \\
& \Sigma_{66}=-H_{6}, \\
& \Sigma_{77}=-H_{7}, \\
& \Sigma_{88}=-H_{8},
\end{aligned}
$$

$$
\begin{aligned}
& \Sigma_{99}=-\gamma^{2} d, \\
& \Sigma_{12}=\sqrt{c_{1}} H_{2}, \\
& \Sigma_{13}=\sqrt{h^{2} c_{1}} H_{3}, \\
& \Sigma_{14}=\sqrt{\rho h c_{1}} H_{4}, \\
& \Sigma_{15}=\sqrt{\rho h c_{1}} H_{5}, \\
& \Sigma_{16}=\sqrt{\frac{1}{2} \rho h^{3} c_{1}} H_{6}, \\
& \Sigma_{17}=\sqrt{\frac{1}{6} \rho h^{3} c_{1}} H_{7}, \\
& \Sigma_{18}=\sqrt{\frac{1}{3} \rho h^{3} c_{1}} H_{8}, \\
& \Sigma_{19}=\bar{P}_{11},
\end{aligned}
$$

others

$$
\Sigma_{i j}=0
$$

then a state feedback $H \infty$ controller in form of (7) exists, such that

(1) the closed-loop system (8) with $u(t)=0$ is FTB;

(2) under given nonzero initial condition, the closed system (8) and (9) guarantees that $J=\int_{0}^{T} z^{T}(s) z(s)-$ $\gamma^{2} \omega^{T}(s) \omega(s)-\gamma^{2}(1 / T) x^{T}(0) S x(0) d s<0$ for all nonzero $\omega(t)$ and $t \in[0, T]$.

The controller gain can be calculated by $K=Y \bar{P}_{11}{ }^{-1}$.

Proof. In preparation for designing, we set $M=\gamma^{2} I, \beta=1$ for (37); then (37) and (38) can be rewritten as follows:

$$
\begin{gathered}
\dot{V}-\alpha V-\gamma^{2} \omega^{T}(t) \omega(t)<\xi^{T}(t) \widehat{\Omega} \xi(t) \\
V(t)<e^{\alpha t} V(0)+\gamma^{2} \int_{0}^{t} e^{\alpha(t-s)} \omega^{T}(s) \omega(s) d s \\
\quad<e^{\alpha T}\left(V(0)+\gamma^{2} d\right) .
\end{gathered}
$$

where

$$
\begin{aligned}
\widehat{\Omega}_{44} & =B_{2}{ }^{T} \frac{1}{2} h^{2}\left(2 W+U_{1}+U_{2}\right) B_{2}-\gamma^{2} I, \\
\widehat{\Omega}_{i j} & =\Omega_{i j}
\end{aligned}
$$

Then, two steps are provided to design the desired controller.

Step 1. Here, we will give some conditions that can guarantee the Ho performance over the finite-time interval under nonzero initial conditions firstly. 
Consider the following inequality:

$$
\begin{aligned}
\dot{V} & -\alpha V+e^{\alpha t} z^{T}(t) z(t)-\gamma^{2} e^{\alpha t} \omega^{T}(t) \omega(t) \\
& <\dot{V}-\alpha V+e^{\alpha T} z^{T}(t) z(t)-\gamma^{2} \omega^{T}(t) \omega(t) \\
& <\xi^{T}(t)\left(\widehat{\Omega}-\Psi^{T}\left(-e^{-\alpha T} I\right)^{-1} \Psi\right) \xi(t) .
\end{aligned}
$$

Noting that $W, U_{1}$, and $U_{2}$ are coupled with $A, B_{1} K$, and $B_{2}$ in $\widehat{\Omega}$, to decouple these terms, the $\widehat{\Omega}$ can be rewritten as

$$
\begin{aligned}
\widehat{\Omega}= & \widetilde{\Omega}-\widetilde{\Psi}^{T}\left(-\frac{1}{2} U_{1}\right)^{-1} \widetilde{\Psi}-\bar{\Psi}^{T}\left(-\frac{1}{2} U_{2}\right)^{-1} \bar{\Psi} \\
& -\widehat{\Psi}^{T}(-W)^{-1} \widehat{\Psi}
\end{aligned}
$$

where

$$
\begin{aligned}
\widetilde{\Omega}_{11}= & A^{T} P_{11}+P_{11} A-\alpha P_{11}+Q_{1}+Q_{2}-4 \rho W \\
& -3 \rho U_{1}-9 \rho U_{2}, \\
\widetilde{\Omega}_{33}= & -8 \rho W+\rho Z_{1}^{T}+\rho Z_{1}-\rho Z_{2}^{T}-\rho Z_{2} \\
& -(1-\mu) Q_{2}, \\
\widetilde{\Omega}_{44}= & -\gamma^{2} I, \\
\widetilde{\Omega}_{13}= & P_{11} B_{1} K-2 \rho W-\rho Z_{1}-\rho Z_{2}, \\
\widetilde{\Omega}_{14}= & P_{11} B_{2}, \\
\widetilde{\Omega}_{34}= & 0,
\end{aligned}
$$

others

$$
\begin{aligned}
& \widetilde{\Omega}_{i j}=\Omega_{i j} \\
& \Psi=\left(\begin{array}{llllllll}
C & 0 & D K & 0 & 0 & 0 & 0 & 0
\end{array}\right) \\
& \widetilde{\Psi}
\end{aligned}
$$

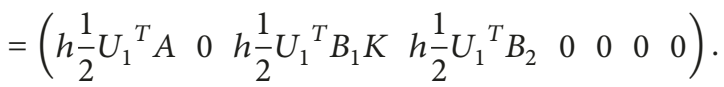

$$
\begin{aligned}
& \bar{\Psi} \\
& =\left(\begin{array}{llllllll}
h \frac{1}{2} U_{2}{ }^{T} A & 0 & h \frac{1}{2} U_{2}{ }^{T} B_{1} K & h \frac{1}{2} U_{2}{ }^{T} B_{2} & 0 & 0 & 0 & 0
\end{array}\right) \\
& \widehat{\Psi}=\left(\begin{array}{llllllll}
h W^{T} A & 0 & h W^{T} B_{1} K & h W^{T} B_{2} & 0 & 0 & 0 & 0
\end{array}\right) .
\end{aligned}
$$

By Schur complement, $\widehat{\Omega}<0$ and $\widehat{\Omega}-\Psi^{T}\left(-e^{-\alpha T} I\right)^{-1} \Psi$ are equivalent to

$$
\Pi=\left(\begin{array}{ccccc}
\widetilde{\Omega} & \widetilde{\Psi}^{T} & \bar{\Psi}^{T} & \widehat{\Psi}^{T} & \Psi^{T} \\
* & -\frac{1}{2} U_{1} & 0 & 0 & 0 \\
* & * & -\frac{1}{2} U_{2} & 0 & 0 \\
* & * & * & -W & 0 \\
* & * & * & * & -e^{-\alpha T} I
\end{array}\right)<0 .
$$

To ensure the proposed conditions satisfy the Ho performance, we set the inequality like

$$
P_{11}-\gamma^{2} S<0
$$

from which we have

$$
\begin{aligned}
\dot{V} & -\alpha V+e^{\alpha t} z^{T}(t) z(t)-\gamma^{2} e^{\alpha t} \omega^{T}(t) \omega(t) \\
& -\gamma^{2} e^{\alpha t} \frac{1}{T} x^{T}(0) S x(0)<\dot{V}-\alpha V+e^{\alpha t} z^{T}(t) z(t) \\
& -\gamma^{2} e^{\alpha t} \omega^{T}(t) \omega(t)-\gamma^{2} e^{\alpha t} \frac{1}{T} x^{T}(0) S x(0) \\
& +e^{\alpha t} \frac{1}{T} x^{T}(0) P_{11} x(0)<\dot{V}-\alpha V+e^{\alpha t} z^{T}(t) z(t) \\
& -\gamma^{2} e^{\alpha t} \omega^{T}(t) \omega(t)+e^{\alpha t} \frac{1}{T} x^{T}(0)\left(P_{11}-\gamma^{2} S\right) x(0) \\
& <0
\end{aligned}
$$

Integrating (58) from 0 to $T$, we can obtain

$$
\begin{aligned}
& \int_{0}^{T} z^{T}(s) z(s) d s-\int_{0}^{T} \gamma^{2} \omega^{T}(s) \omega(s) d s \\
& \quad-\gamma^{2} x^{T}(0) S x(0)<-\int_{0}^{T}\left(e^{-\alpha t} \dot{V}-\alpha e^{-\alpha t} V\right) d s<0
\end{aligned}
$$

so conditions (43), (44), (57), and (58) with the inequalities as follows can ensure that systems (17) and (18) satisfy the $H \infty$ performance:

$$
\begin{aligned}
c_{1} & \theta_{2}+h^{2} c_{1} \theta_{3}+\rho h c_{1} \theta_{4}+\rho h c_{1} \theta_{5}+\frac{1}{2} \rho h^{3} c_{1} \theta_{6} \\
& \quad+\frac{1}{6} \rho h^{3} c_{1} \theta_{7}+\frac{1}{3} \rho h^{3} c_{1} \theta_{8}+\gamma^{2} d<e^{-\alpha T} c_{2} \theta_{1} \\
0 & <\theta_{1} I<P_{11}<\theta_{2} I, \\
0 & <P_{22}<\theta_{3} I, \\
0 & <Q_{1}<\theta_{4} I, \\
0 & <Q_{2}<\theta_{5} I \\
0 & <W<\theta_{6} I, \\
0 & <U_{1}<\theta_{7} I, \\
0 & <U_{2}<\theta_{8} I .
\end{aligned}
$$


Step 2. In this step, the approach to design the gain matrix $K$ of the controller is provided in forms of LMIs, which can be calculated expediently.
To linearize the nonlinear terms in (57), we make it multiply by the following matrix from both left and right sides firstly:

$$
\operatorname{diag}\left\{\begin{array}{llllllllllll}
P_{11}^{-1} & P_{11}^{-1} & P_{11}^{-1} & I & P_{11}^{-1} & P_{11}^{-1} & P_{11}^{-1} & P_{11}^{-1} & U_{1}^{-1} & U_{2}^{-1} & W^{-1} & I
\end{array}\right\}
$$

Let us do the definitions as follows:

$$
\begin{aligned}
& \bar{P}_{11}=P_{11}^{-1}, \\
& \bar{Q}_{1}=P_{11}^{-1} Q_{1} P_{11}^{-1}, \\
& \bar{Q}_{2}=P_{11}^{-1} Q_{2} P_{11}^{-1}, \\
& \bar{W}=P_{11}^{-1} W P_{11}^{-1}, \\
& \bar{U}_{1}=P_{11}^{-1} U_{1} P_{11}^{-1}, \\
& \bar{U}_{2}=P_{11}^{-1} U_{2} P_{11}^{-1}, \\
& \bar{P}_{22}=P_{11}^{-1} P_{22} P_{11}^{-1}, \\
& \bar{Z}_{1}=P_{11}^{-1} Z_{1} P_{11}^{-1}, \\
& \bar{Z}_{2}=P_{11}^{-1} Z_{2} P_{11}^{-1}, \\
& Y=K P_{11}^{-1} .
\end{aligned}
$$

so the inequality $\bar{\Pi}<0$ can guarantee $\Pi<0$ where

$$
\begin{aligned}
\bar{\Pi}_{99} & =-\frac{1}{2} \bar{P}_{11} \bar{U}_{1}^{-1} \bar{P}_{11}, \\
\bar{\Pi}_{10,10} & =-\frac{1}{2} \bar{P}_{11} \bar{U}_{2}^{-1} \bar{P}_{11}, \\
\bar{\Pi}_{11,11} & =-\bar{P}_{11} \bar{W}^{-1} \bar{P}_{11},
\end{aligned}
$$

others

$$
\bar{\Pi}_{i j}=\widetilde{\Pi}_{i j}
$$

Then, based on the inequalities $-(1 / 2) \bar{P}_{11} \bar{U}_{1}^{-1} \bar{P}_{11} \leq$ $(1 / 2) \bar{U}_{1}-\bar{P}_{11},-(1 / 2) \bar{P}_{11} \bar{U}_{2}^{-1} \bar{P}_{11} \leq(1 / 2) \bar{U}_{2}-\bar{P}_{11}$, $-\bar{P}_{11} \bar{W}^{-1} \bar{P}_{11} \leq \bar{W}-2 \bar{P}_{11}$, condition (57) can be converted into (41) in form of LMIs.

Accordingly, similar to the above-mentioned procedures, (61) can be transformed into (42) with the Schur complement and the definition of $H_{i}=P_{11}^{-1} \theta_{i} P_{11}^{-1}(i=1,2, \cdots, 8)$.

Therefore, conditions (41) to (45) can obtain the controller in the form of (7) guaranteeing the desired performance. This completes the proof.

Remark 16. The Ho performance with nonzero initial condition is studied in this literature. In a practical view, it is hard to suffer the worst-case over a finite time for surface vessel with nonzero initial condition and it will reduce the conservatism caused by the assumption of zero initial condition. Therefore, it is reasonable to introduce such $H \infty$ performance index to handle the problem of finite-time $H \infty$ control for ship subject to exogenous signals.

Remark 17. It is challenging to achieve the result satisfying the control objective while it is very different from the standard $H \infty$ control because a weighting diagonal matrix is introduced, especially to establish the form of LMIs. In the proof of Theorem 15, inequality (58) is introduced to ensure the $H \infty$ performance with nonzero initial condition and make it convenient to construct the conditions in form of LMIs.

Remark 18. By introducing some new additional matrix variables, Cone Complementarity Linearization algorithm (CCL) [32] can be utilized to linearize the nonlinear terms as an alternative method. It will reduce the conservatism at the expense of increasing the computational burden and the number of decision variables.

\section{Simulations}

4.1. Simulation Settings. To demonstrate the effectiveness and superiority of the robust finite-time $H \infty$ control scheme, simulation studies are conducted on the parameters of the ship CyberShip II [33], which is a 1:70 scale-replica of a supply ship whose main parameters are shown in Table 1 . In addition, we choose the time-varying function like $h(t)=3 \sin (t / 6-$ $\pi / 6)$ to describe the time delay occurred in the input signal.

Unknown external disturbances $\omega(t)$ with significantly large magnitude and high frequency, which can be roughly considered as complex environment including ocean winds, waves, and currents, are governed by [34]

$$
\omega(t)=\left(\begin{array}{c}
9 \sin \left(0.1 \pi t-\frac{\pi}{5}\right) \\
6 \sin \left(0.3 \pi t+\frac{\pi}{6}\right) \\
3 \sin \left(0.2 \pi t+\frac{\pi}{3}\right)
\end{array}\right)
$$

with initial conditions $\eta(0)=\left(\begin{array}{lll}1 & 1 & 0.1\end{array}\right)$ and $v(0)=$ $\left(\begin{array}{lll}0.8 & 0.6 & 0.1\end{array}\right)$.

4.2. Numerical Simulations. We choose $\gamma=1.3, T=10, S=$ $0.3 * I, c_{1}=3.02, c_{2}=10, \alpha=0.001$. By using Theorem 15 , the control gain $K$ is calculated by LMIs (41) to (45): 
TABle 1: The main parameters of the CyberShip II.

\begin{tabular}{lccccc}
\hline$m$ & 23.8000 & $X_{\dot{u}}$ & -2.0 & $X_{u}$ & -0.7225 \\
$I_{z}$ & 1.7600 & $Y_{\dot{v}}$ & -10.0 & $Y_{v}$ & -0.8612 \\
$x_{g}$ & 0.0460 & $Y_{\dot{r}}$ & -0.0 & $Y_{r}$ & 0.1079 \\
$N_{\dot{r}}$ & -1.0 & $N_{r}$ & 0.5 & \\
\hline
\end{tabular}
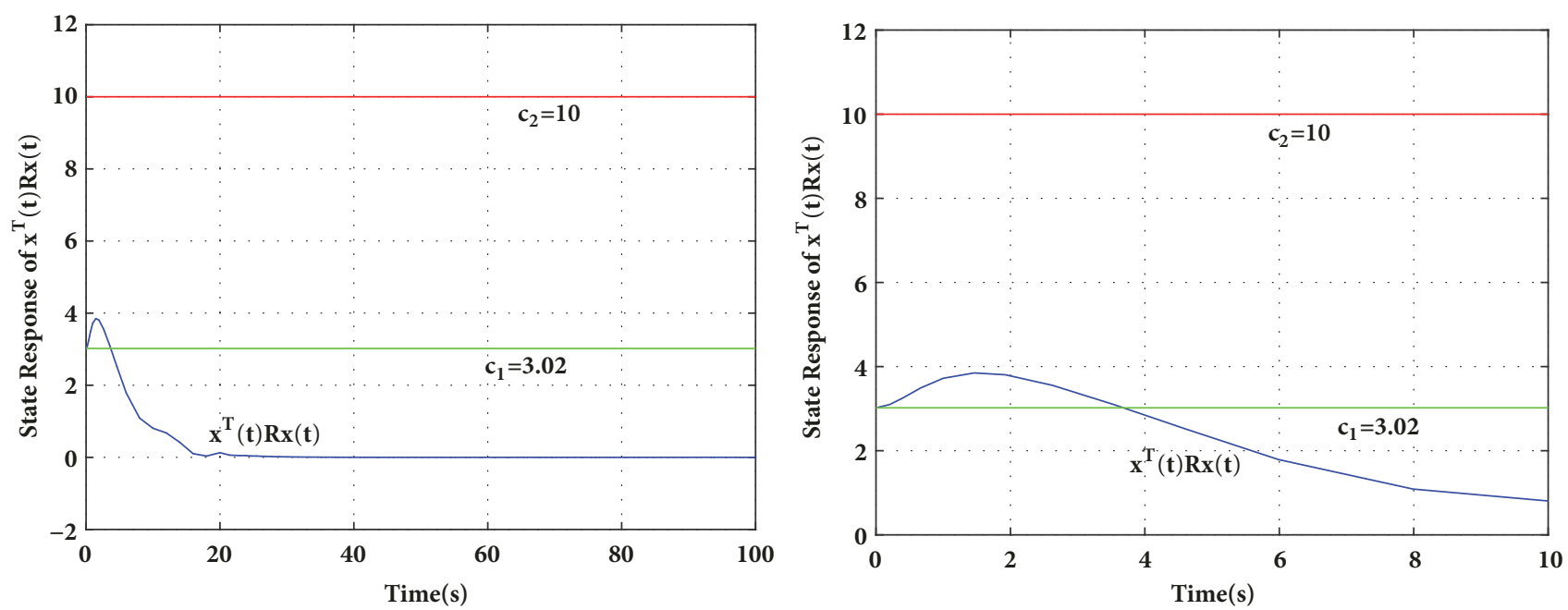

Figure 1: State response $x^{T}(t) R x(t)$ of DP system.

$$
K=\left(\begin{array}{cccccc}
-3.2743 & 0 & 0 & -31.3907 & 0 & 0 \\
0 & -4.2784 & -0.1572 & 0 & -41.2136 & -1.2982 \\
0 & -0.1389 & -0.3497 & 0 & -1.3362 & -3.3091
\end{array}\right)
$$

To demonstrate the superiority and validity, three scenarios as comparative cases are provided here. It should be pointed out that the results within 100s are also provided to illustrate the steady-state performance.

Case 1 (with different $S$ ). For comparison purposes, we set $S$ with different value to demonstrate the effect of $S$ on state response. Without loss of generality, we set $S=0.5 * I$ and $S=0.1 * I$, respectively.

Case 2 (Ho with zero initial condition]). This scenario will show that the control law proposed in this paper can achieve a better transient performance and disturbance rejection capability over traditional $H \infty$ control approach for dynamic positioning system conducted in [11].

Case 3 (a control design based on existing methods). Based on the approach in [17] applied for discrete-time system, a control law for dynamic positioning ship with input delay is proposed to illustrate the less conservatism of our designing approach in practical engineering subsequently.

At first, the effectiveness of the control law we designed is confirmed from Figures 1-6. Figure 1 shows the state response of $x^{T}(t) R x(t)$. Obviously, from that curve we can see that the value of $x^{T}(t) R x(t)$ is far below the value of $c_{2}=10$ in the presence of disturbance as in Figure 2, which means the finite-time full-state feedback controller designed can guarantee the DP system (8) (9) FTB with (3.02 $\left.10 \quad d \quad \begin{array}{lll}10 & 3 & I\end{array}\right)$ and have less conservatism, where we set $d=\int_{0}^{T} \omega(t)^{T} \omega(t) d t$. Meanwhile, the control law can stabilize the dynamic positioning ship with minimum steadystate error in 100s and the control input will be small enough as shown in Figure 6. From Figures 3-5, we can find that all states in DP system are within a small range. As shown in Figures 2-4, the angle and the angular velocity in yaw have changed within $0.1^{\circ}$, which means that the heading of the vessel stays in a fixed direction. Furthermore, the velocities in surge and sway are low enough, which illustrates that assumption 2 is reasonable and it meets the requirement of stabilization control for DP system.

To demonstrate the effect of $S$, state responses of systems with different $S$ are exhibited in Figures 7-9. As the value of $S$ decreases, the restriction for $P_{11}$ is more exclusive and the robustness is stronger during preset time interval as in Figures 7 and 8 while the required control input will increase dramatically as in Figure 9. When the time oversteps the 


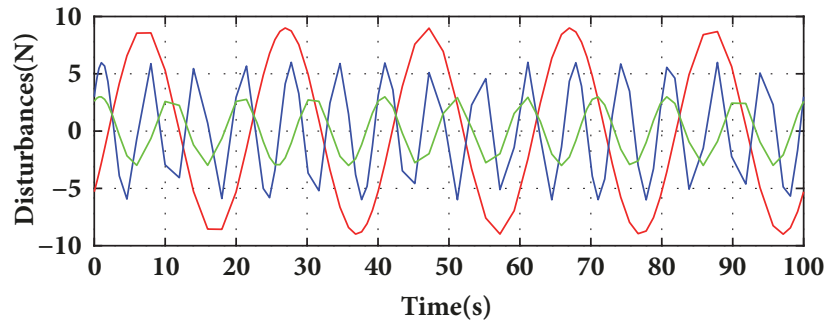

$$
\begin{array}{r}
\mathbf{X} \\
-\mathbf{Y} \\
-\psi
\end{array}
$$

FIgURE 2: Disturbance in surge, sway, and yaw.
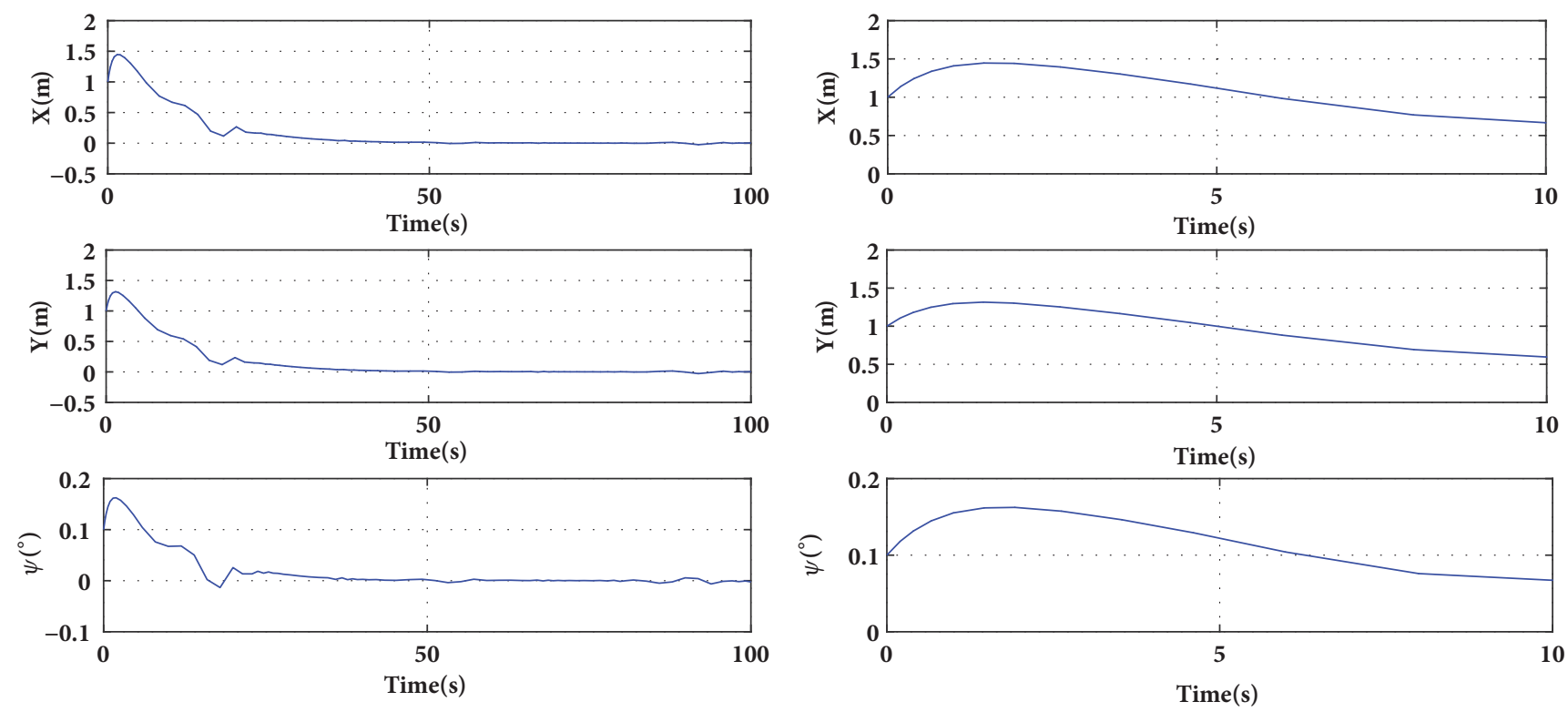

FIGURE 3: State responses of position and orientation for DP System.
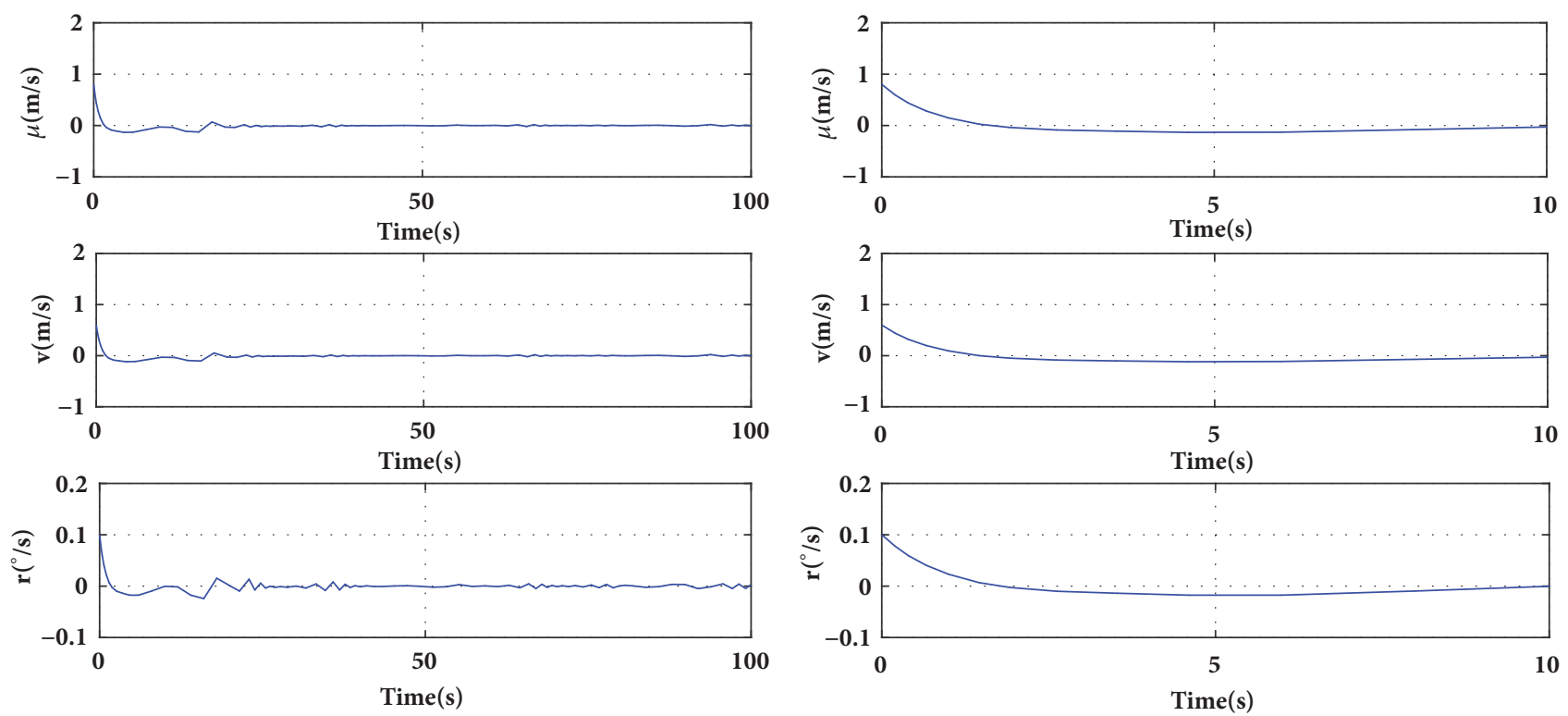

FIGURE 4: State responses of velocities for DP system. 


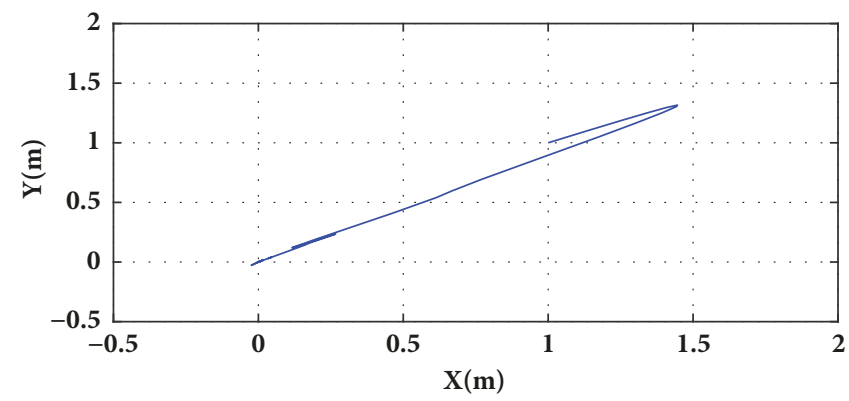

FIGURE 5: Trajectory of the ship's horizontal motion.
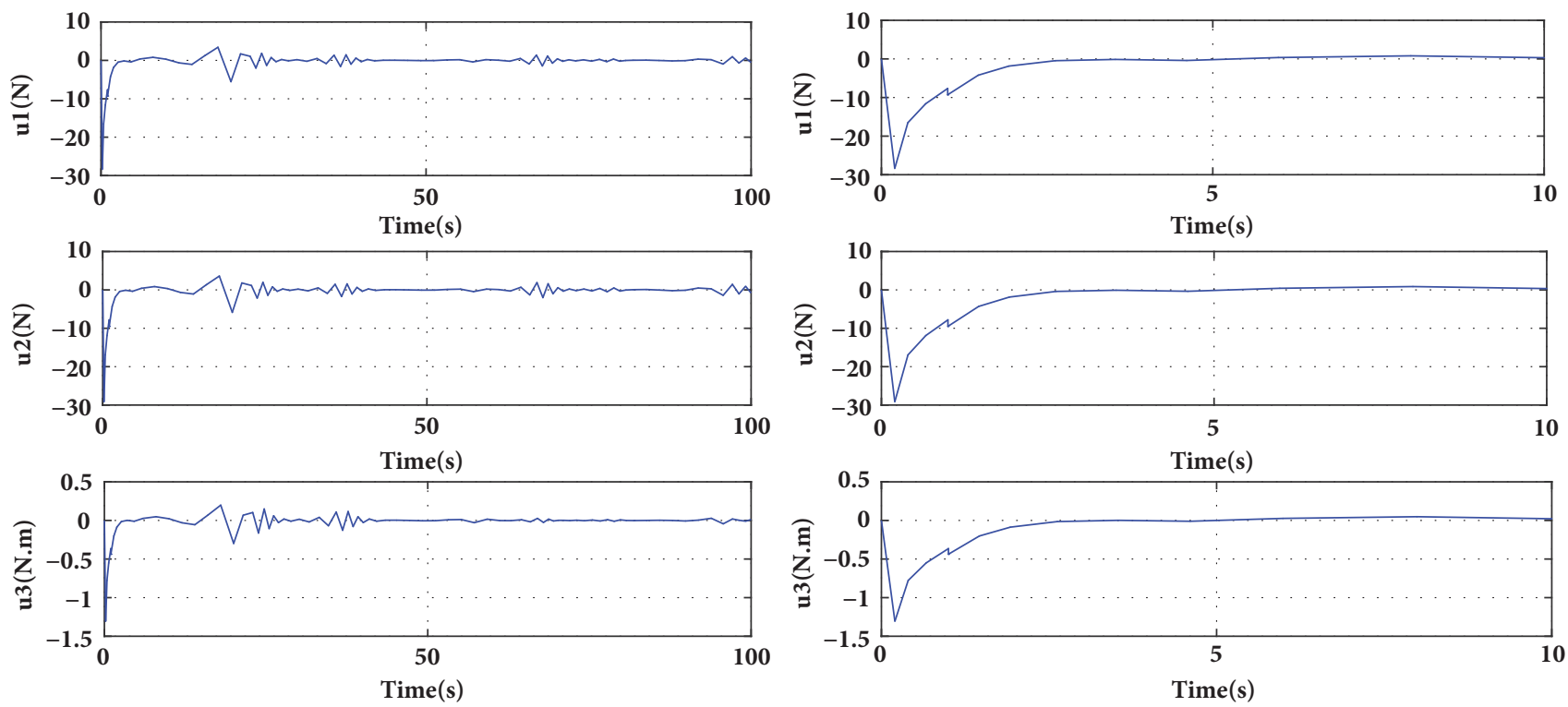

Figure 6: Control inputs.
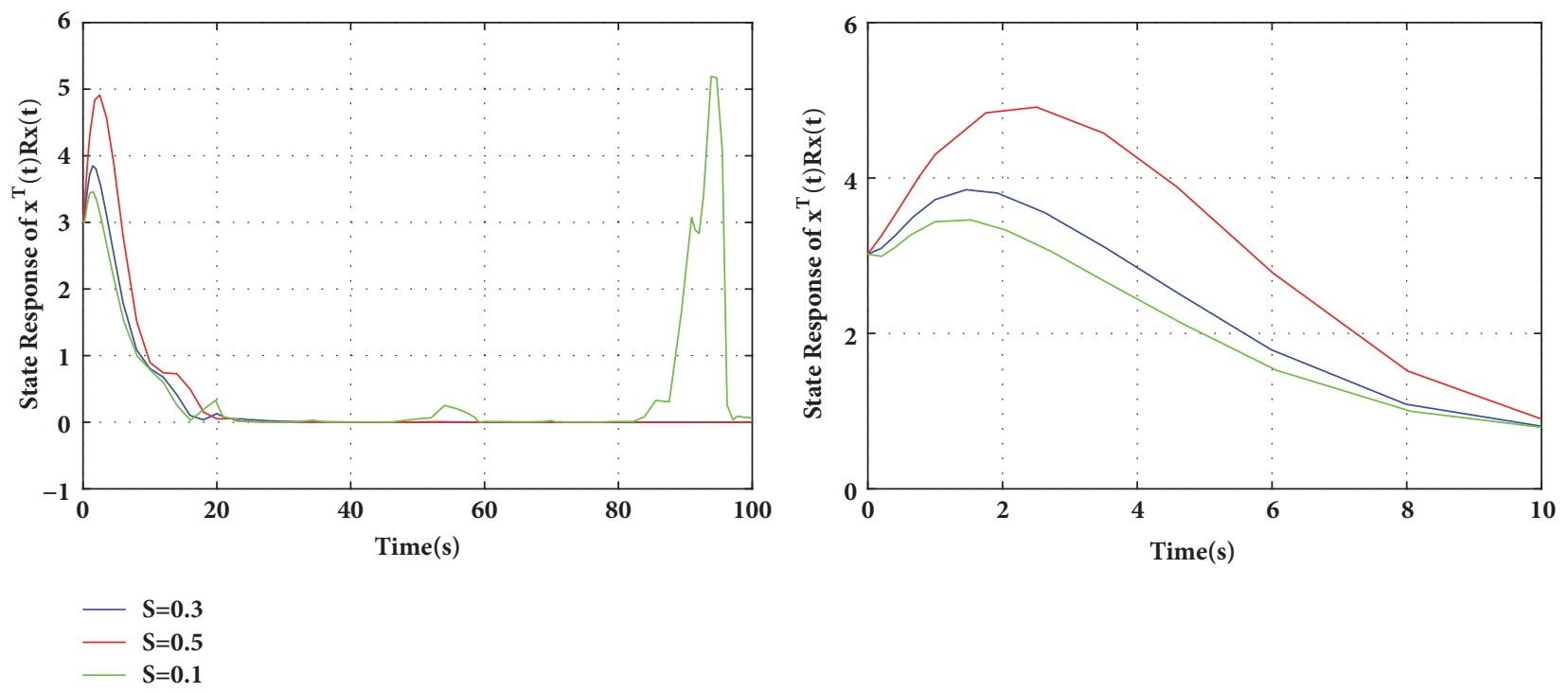

FIGURE 7: State response $x^{T}(t) R x(t)$ with different $S$. 

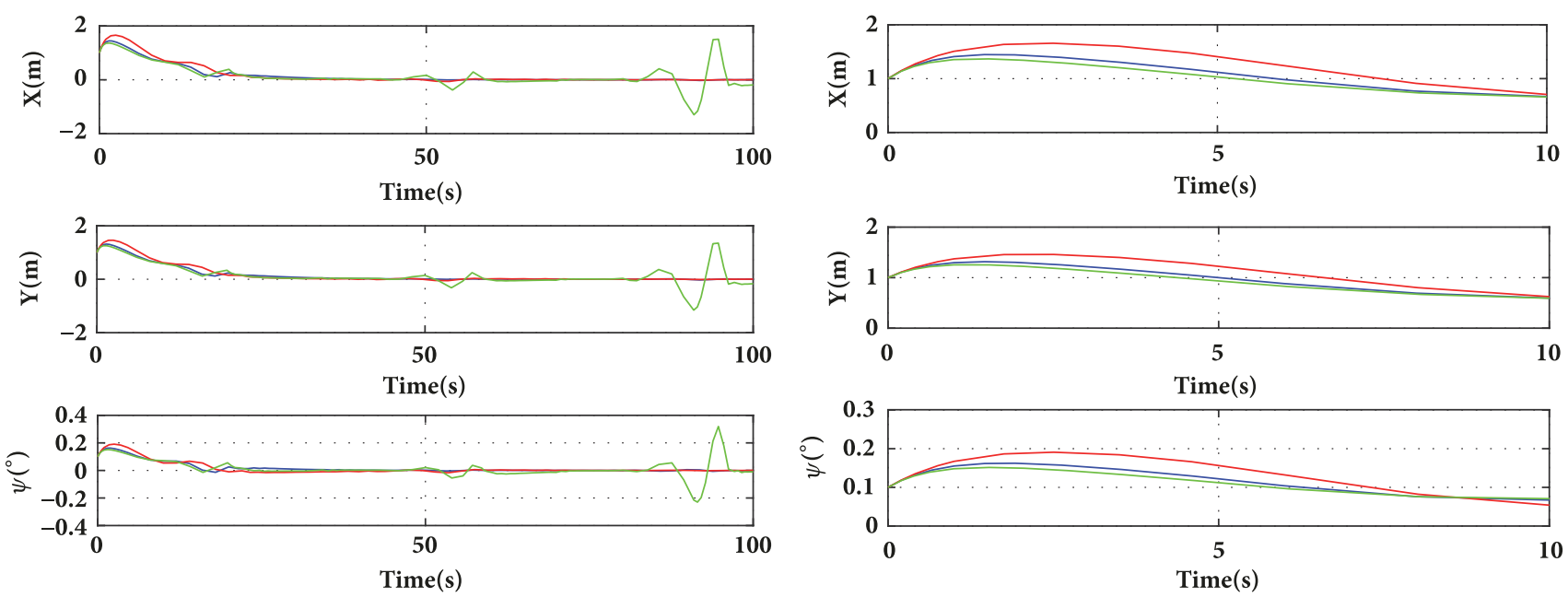

$S=0.3$
$S=0.5$
$S=0.1$

FIGURE 8: State responses of position and orientation with different $S$.
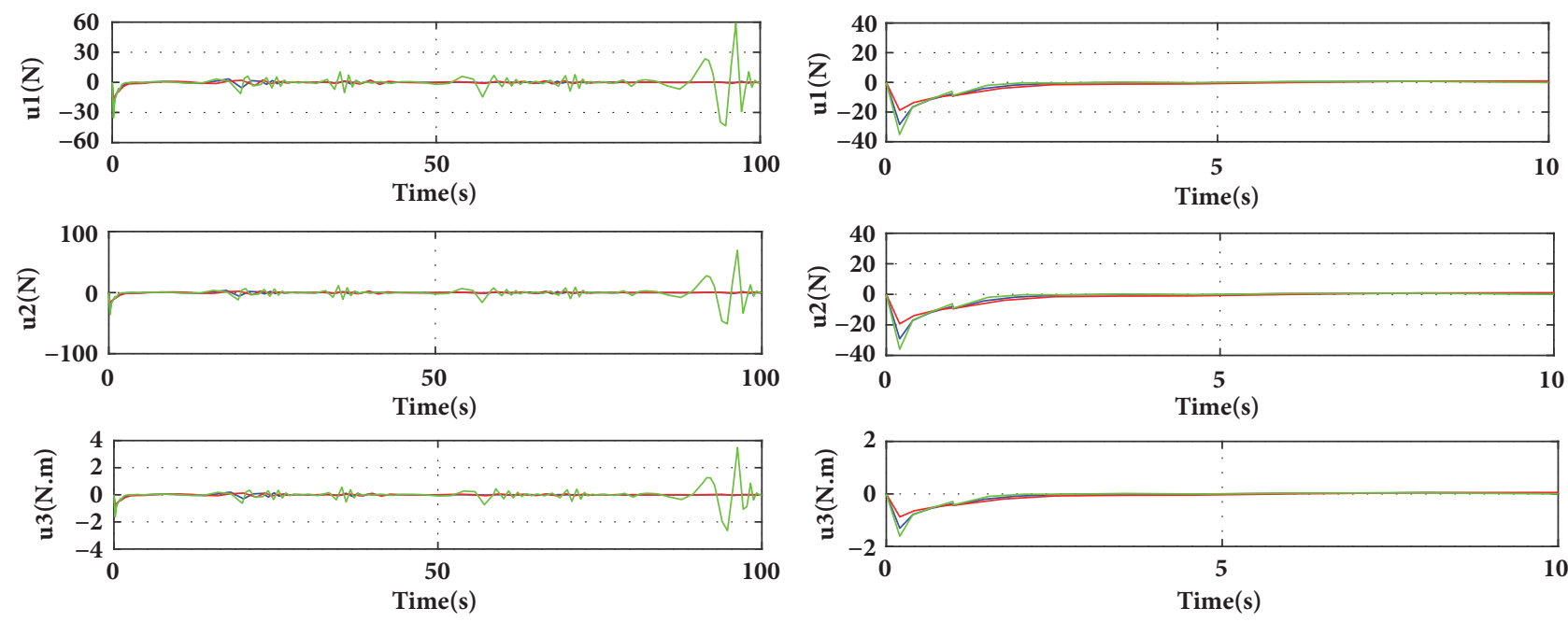

$S=0.3$
$S=0.5$
$S=0.1$

FIGURE 9: Control input with different $S$.

preset time interval, all the states can be guaranteed within the given upper bound as before.

As shown in Figures 10-12, the overconservatism of traditional $H \infty$ control approach applied in DP ship is solved by the $H \infty$ control strategy with nonzero initial condition proposed in this article. As is well known, the classical $H \infty$ control law is designed under the so-called worst-case norm while the performance of antidisturbance and transient is sacrificed to some degree. From Figures 10 and 11 we can see that the state response can be controlled in a smaller range and stabilized in a shorter time with a lower overshoot shown in Figure 12. Hence, a conclusion can be drawn that the control law we present can achieve a better disturbance rejection capability and transient performance while the matter of overconservatism can be resolved at the same time.

Simulation results of Theorem 15 and Case 3 are shown in Figures 13 and 14 to show the better performance of the method we proposed. We can find that the controller based on [17] and $H \infty$ control approach present in this literature can make all states of system within a small enough region. However, compared with the controller we constructed, the state response has higher overshoot which will affect the control precision and transient performance in practical engineering. Based on the above simulation results, the developed controller can achieve a higher accuracy and 


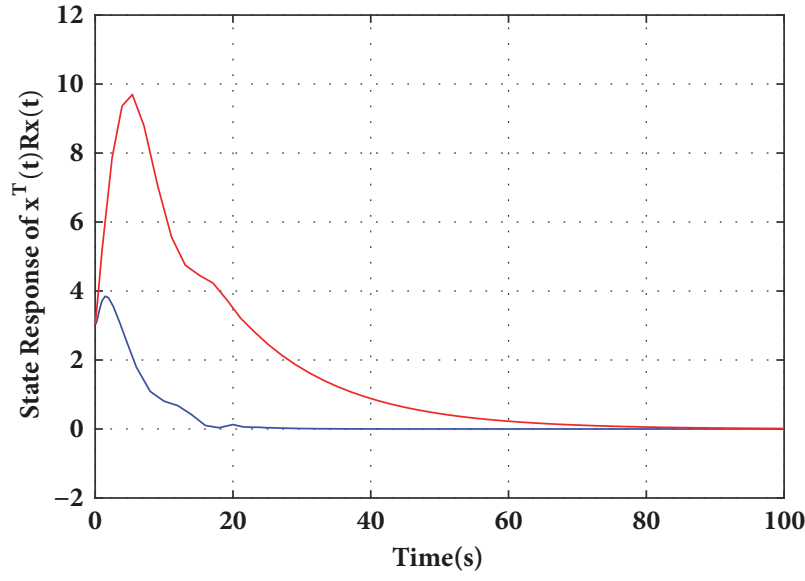

- Theorem 15

- Case 2

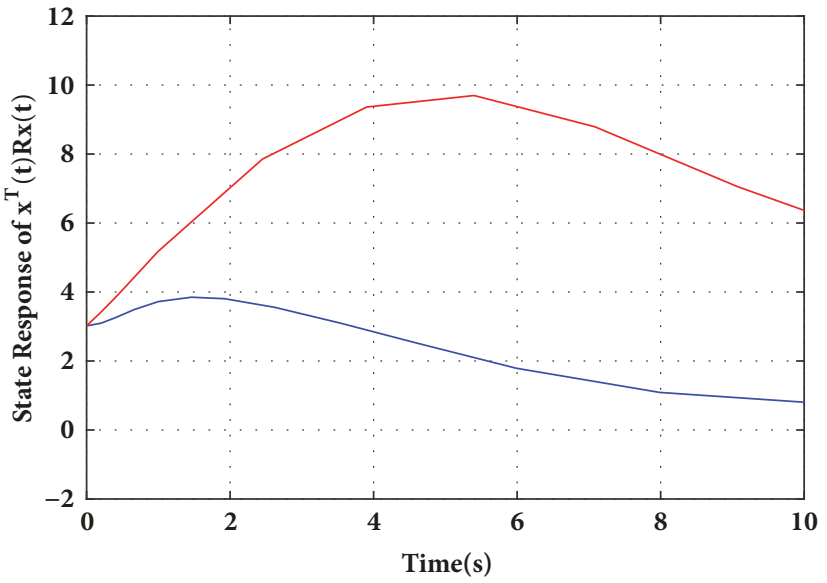

— Theorem 15

- Case 2

FIGURE 10: State response $x^{T}(t) R x(t)$ of Theorem 15 and Case 2.
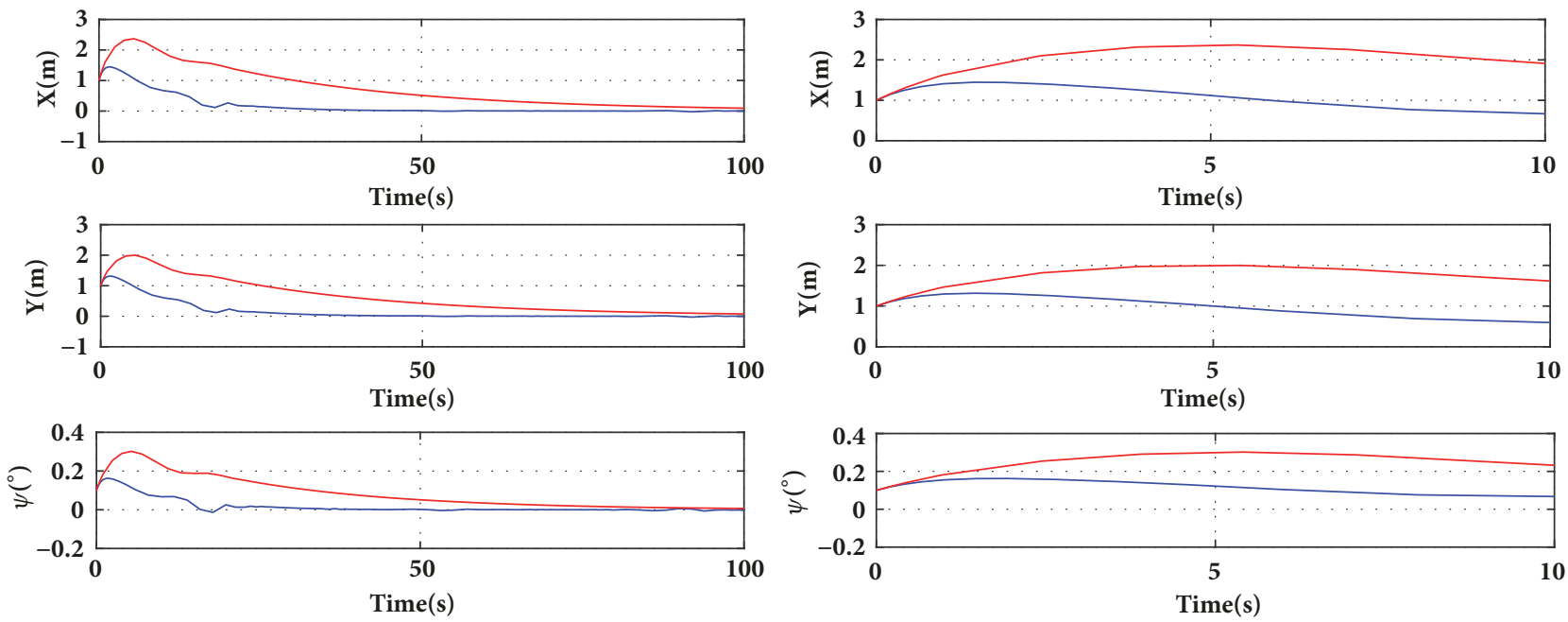

- Theorem 15

- Case 2

FIgure 11: Position and orientation of Theorem 15 and Case 2.

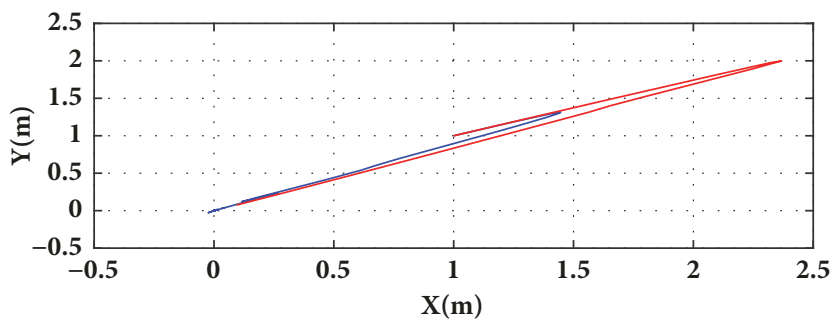

\section{— Theorem 15}

- Case 2

FIGURE 12: Trajectory of the ship's horizontal motion of Theorem 15 and Case 2. 

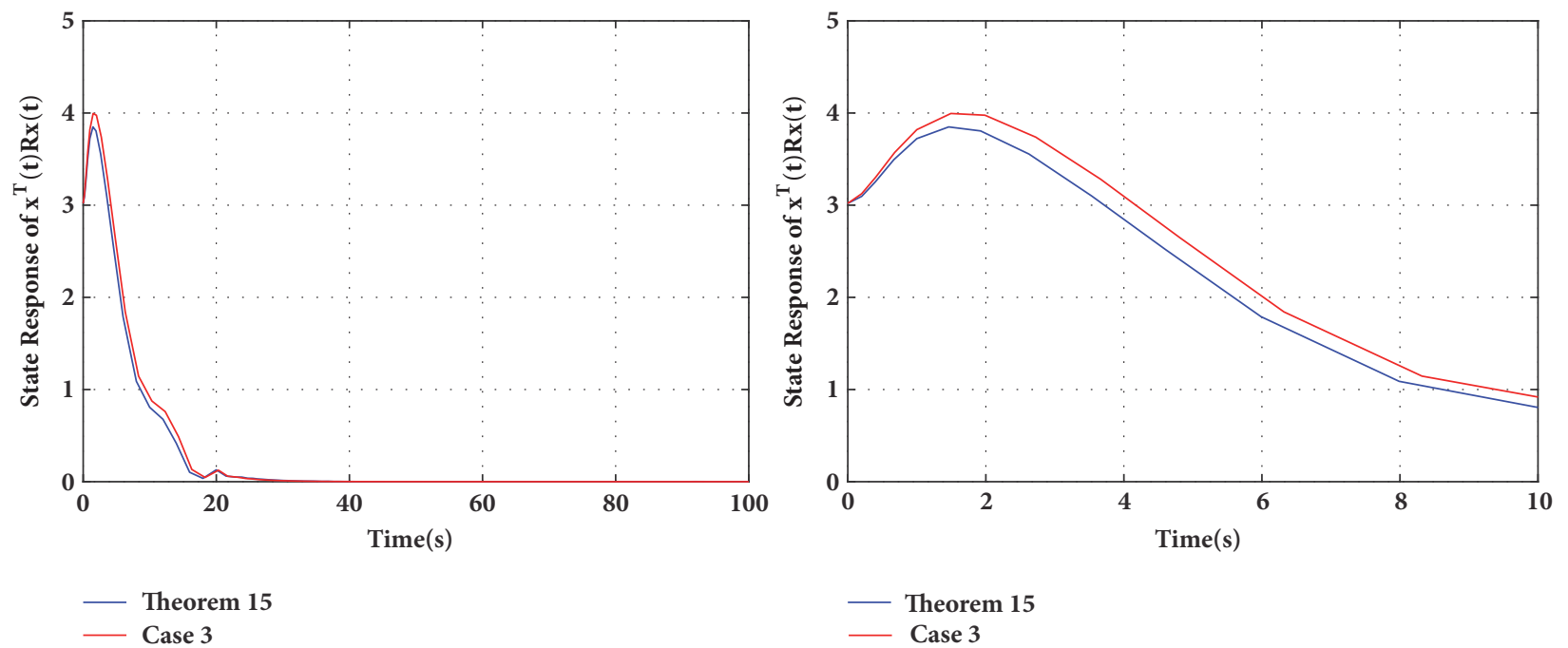

Figure 13: State response $x^{T}(t) R x(t)$ of Theorem 15 and Case 3.
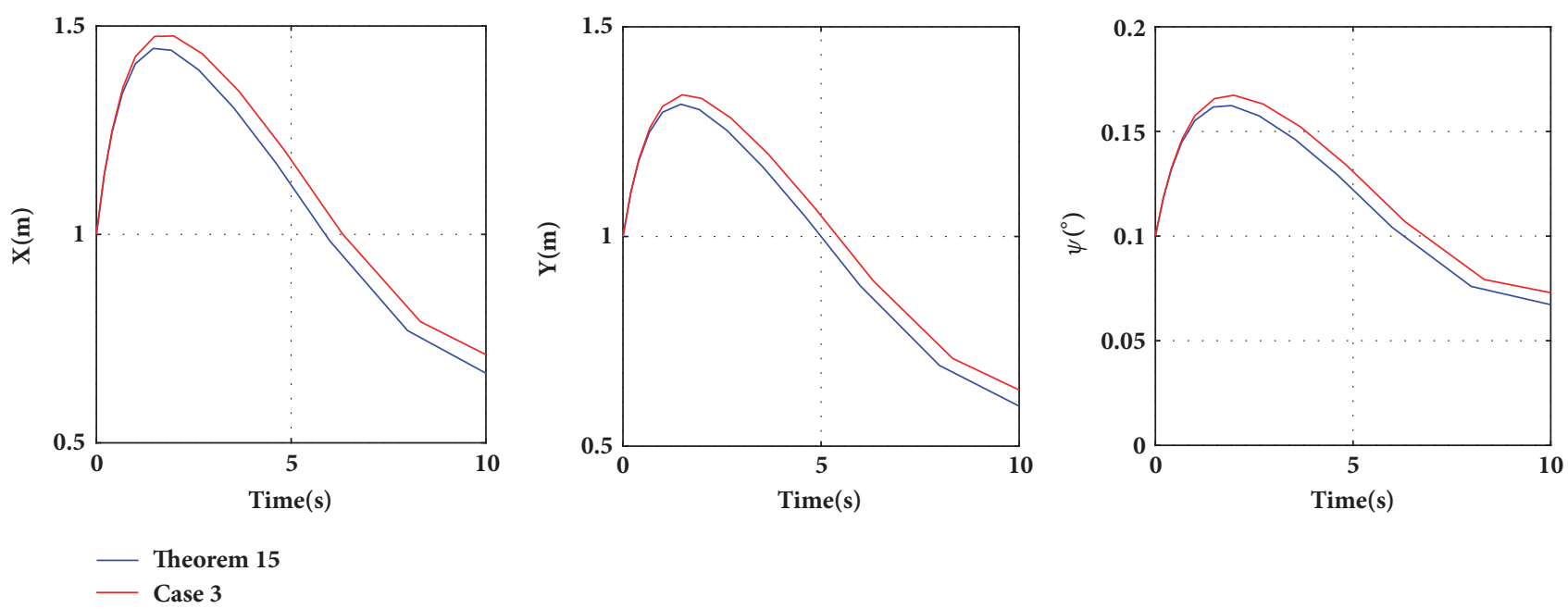

FIgURE 14: Position and orientation of Theorem 15 and Case 3.

lower overshoot, which means that it is less conservative in comparison with existing result.

\section{Conclusion}

In this paper, the robust finite-time $H \infty$ control with transients for DP system with input delay, maintaining all states of ocean surface vessel into a given threshold over a fixed time interval in presence of time-varying disturbance, is proposed. Based on a novel augmented LKLF with triple integral terms and a method combining the Wirtinger inequality and reciprocally convex approach, a less conservative result is derived. In particular, a $H \infty$ performance index with nonzero initial condition, attenuating the overconservatism caused by the assumption of zero initial condition and enhancing the transient performance of ship subject to external disturbance, is adopted. The obtained result is formulated in terms of LMIs which can be easily solved by the standard numerical software. Finally, a numerical simulation for a ship is proposed to verify the effectiveness and less conservatism of the controller we designed. In the future, the output feedback control can be developed for the ocean surface vessel to avoid the use of the vessel-frame surge, sway, and yaw velocities.

\section{Data Availability}

All the data supporting the conclusions of the study have been provided in Simulations and readers can access these data in $[33,34]$.

\section{Conflicts of Interest}

The authors declare that there are no conflicts of interest related to this paper.

\section{Acknowledgments}

This research was partially supported by the National Science Technology Support Program of China (Project no. 51609046). 


\section{References}

[1] M. Chen, B. Jiang, and R. Cui, "Actuator fault-tolerant control of ocean surface vessels with input saturation," International Journal of Robust \& Nonlinear Control, vol. 26, no. 3, pp. 542564, 2016.

[2] J. Du, X. Hu, and Y. Sun, "Robust dynamic positioning of ships with disturbances under input saturation," Automatica, vol. 73, pp. 207-214, 2016.

[3] H. Ashrafiuon, K. R. Muske, L. C. McNinch, and R. A. Soltan, "Sliding-mode tracking control of surface vessels," IEEE Transactions on Industrial Electronics, vol. 55, no. 11, pp. 40044012, 2008.

[4] W. Si and X. Dong, "Neural prescribed performance control for uncertain marine surface vessels without accurate initial errors," Mathematical Problems in Engineering, Art. ID 2618323, 11 pages, 2017.

[5] T. D. Nguyen, A. J. Sørensen, and S. T. Quek, "Design of hybrid controller for dynamic positioning from calm to extreme sea conditions," Automatica, vol. 43, no. 5, pp. 768-785, 2007.

[6] J. Du, X. Hu, H. Liu, and C. L. Chen, "Adaptive robust output feedback control for a marine dynamic positioning system based on a high-gain observer," IEEE Transactions on Neural Networks and Learning Systems, vol. 26, no. 11, pp. 2775-2786, 2015.

[7] H. Katayama, "Nonlinear sampled-data stabilization of dynamically positioned ships," IEEE Transactions on Control Systems Technology, vol. 18, no. 2, pp. 463-468, 2010.

[8] K. J. Astrom and C. G. Kallström, "Identification of ship steering dynamics," Automatica, vol. 12, no. 1, pp. 9-22, 1976.

[9] Z. Lei and C. Guo, "Disturbance rejection control solution for ship steering system with uncertain time delay," Ocean Engineering, vol. 95, pp. 78-83, 2015.

[10] J. C. Doyle, K. Glover, P. P. Khargonekar, and B. A. Francis, "State-space solutions to standard $\mathrm{H}-2$ and $\mathrm{H} \infty$ control problems," IEEE Transactions on Automatic Control, vol. 34, no. 8, pp. 831-847, 1989.

[11] M. Katebi, M. Grimble, and Y. Zhang, "Ho robust control design for dynamic ship positioning," IEE Proceedings - Control Theory and Applications, vol. 144, no. 2, pp. 110-120, 1997.

[12] D. V. Balandin and M. M. Kogan, "LMI-based Ho-optimal control with transients," International Journal of Control, vol. 83, no. 8, pp. 1664-1673, 2010.

[13] Z. Feng, J. Lam, S. Xu, and S. Zhou, "Ho Control with Transients for Singular Systems," Asian Journal of Control, vol. 18, no. 3, pp. 817-827, 2016.

[14] P. P. Khargonekar, K. M. Nagpal, and K. R. Poolla, "Ho control with transients," SIAM Journal on Control and Optimization, vol. 29, no. 6, pp. 1373-1393, 1991.

[15] Z. Zhang, Z. Zhang, and H. Zhang, "Finite-time stability analysis and stabilization for uncertain continuous-time system with time-varying delay," Journal of The Franklin Institute, vol. 352, no. 3, pp. 1296-1317, 2015.

[16] J. Cheng, S. Chen, Z. Liu, H. Wang, and J. Li, "Robust finitetime sampled-data control of linear systems subject to random occurring delays and its application to Four-Tank system," Applied Mathematics and Computation, vol. 281, pp. 55-76, 2016.

[17] W. Kang, S. Zhong, K. Shi, and J. Cheng, "Finite-time stability for discrete-time system with time-varying delay and nonlinear perturbations," ISA Transactions ${ }^{\circledR}$, vol. 60, pp. 67-73, 2016.

[18] T. I. Fossen, Handbook of Marine Craft Hydrodynamics and Motion Control, John Wiley \& Sons, New York, USA, 2011.
[19] D. Zhao, F. Ding, L. Zhou, W. Zhang, and H. Xu, "Robust H-infinity Control of Neutral System with Time-Delay for Dynamic Positioning Ships," Mathematical Problems in Engineering, vol. 2015, Article ID 976925, 11 pages, 2015.

[20] F. Amato, M. Ariola, and C. Cosentino, "Finite-time stability of linear time-varying systems: analysis and controller design," Institute of Electrical and Electronics Engineers Transactions on Automatic Control, vol. 55, no. 4, pp. 1003-1008, 2010.

[21] L. Sun and Z. Zheng, "Finite-time sliding mode trajectory tracking control of uncertain mechanical systems," Asian Journal of Control, vol. 19, no. 1, pp. 399-404, 2017.

[22] P. Shi, Y. Zhang, and R. Agarwal, "Stochastic finite-time state estimation for discrete time-delay neural networks with Markovian jumps," Neurocomputing, vol. 151, part 1, pp. 168-174, 2014.

[23] L. Zhang, S. Wang, H. R. Karimi, and A. Jasra, "Robust FiniteTime Control of Switched Linear Systems and Application to a Class of Servomechanism Systems," IEEE/ASME Transactions on Mechatronics, vol. 20, no. 5, pp. 2476-2485, 2015.

[24] L. Wu, P. Shi, and H. Gao, "State estimation and slidingmode control of Markovian jump singular systems," Institute of Electrical and Electronics Engineers Transactions on Automatic Control, vol. 55, no. 5, pp. 1213-1219, 2010.

[25] Y. Q. Zhang, P. Shi, S. K. Nguang, and Y. D. Song, "Robust finitetime Ho control for uncertain discrete-time singular systems with Markovian jumps," IET Control Theory \& Applications, vol. 8, no. 12, pp. 1105-1111, 2014.

[26] S. Wang, T. Shi, L. Zhang, A. Jasra, and M. Zeng, "Extended finite-time $\mathrm{H} \infty \mathrm{o}$ control for uncertain switched linear neutral systems with time-varying delays," Neurocomputing, vol. 152, pp. 377-387, 2015.

[27] J. Hu, G. Sui, S. Du, and X. Li, "Finite-time stability of uncertain nonlinear systems with time-varying delay," Mathematical Problems in Engineering, Article ID 2538904, 9 pages, 2017.

[28] A. Seuret and F. Gouaisbaut, "Wirtinger-based integral inequality: application to time-delay systems," Automatica, vol. 49, no. 9, pp. 2860-2866, 2013.

[29] Z. Li, Y. Bai, C. Huang, and Y. Cai, "Novel delay-partitioning stabilization approach for networked control system via Wirtinger-based inequalities," ISA Transactions ${ }^{\circledR}$, vol. 61, pp. 75-86, 2016.

[30] P. Park, J. W. Ko, and C. Jeong, "Reciprocally convex approach to stability of systems with time-varying delays," Automatica, vol. 47, no. 1, pp. 235-238, 2011.

[31] K. Gu, V. L. Kharitonov, and J. Chen, Stability of Time Delay Systems, Birkhäuser, Boston, Mass, USA, 2003.

[32] L. El Ghaoui, F. Oustry, and M. AitRami, "A cone complementarity linearization algorithm for static output-feedback and related problems," Institute of Electrical and Electronics Engineers Transactions on Automatic Control, vol. 42, no. 8, pp. 1171-1176, 1997.

[33] R. Skjetne, T. I. Fossen, and P. Kokotovic, "Adaptive maneuvering, with experiments, for a model ship in a marine control laboratory," Automatica, vol. 41, no. 2, pp. 289-298, 2005.

[34] N. Wang, C. Qian, J.-C. Sun, and Y.-C. Liu, "Adaptive Robust Finite-Time Trajectory Tracking Control of Fully Actuated Marine Surface Vehicles," IEEE Transactions on Control Systems Technology, vol. 24, no. 4, pp. 1454-1462, 2016. 


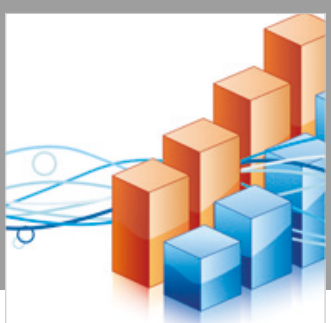

Advances in

Operations Research

\section{-n-m}
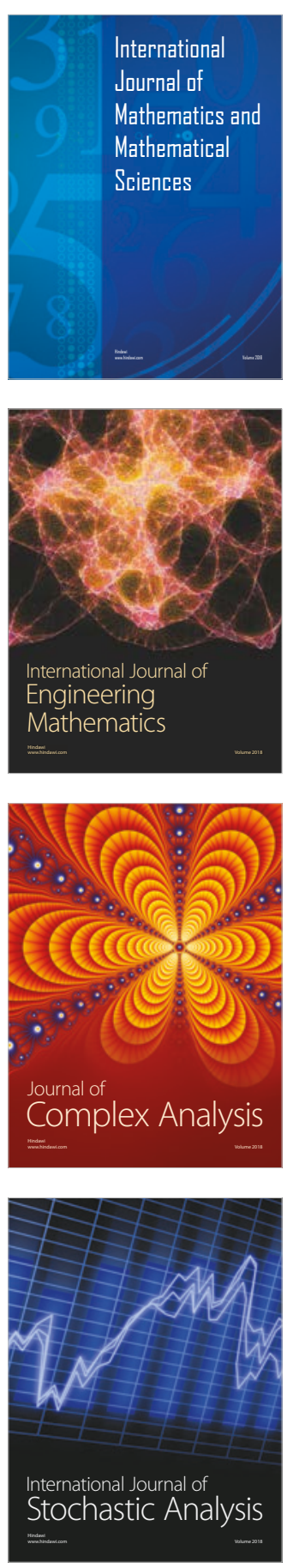
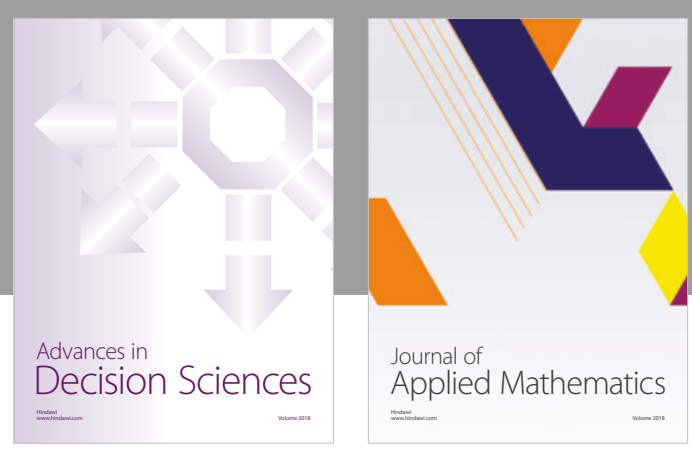

Journal of

Applied Mathematics
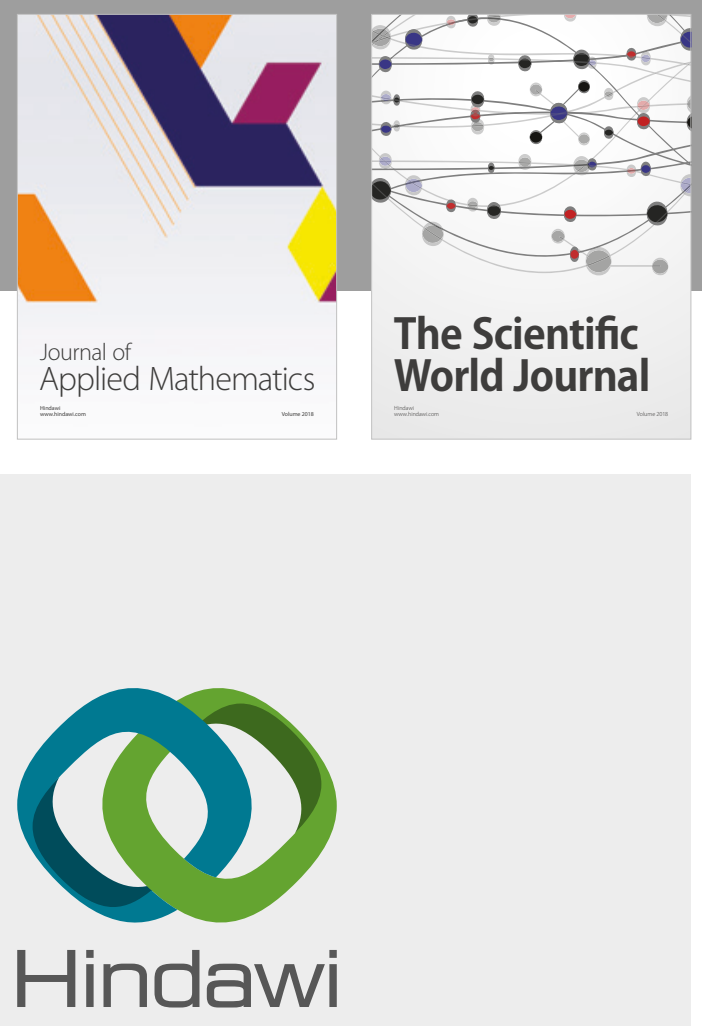

Submit your manuscripts at

www.hindawi.com

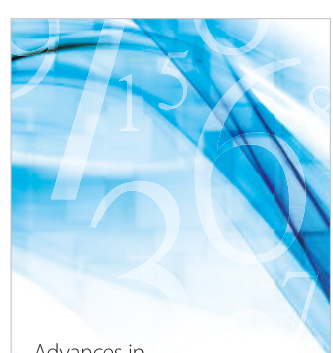

Advances in
Numerical Analysis
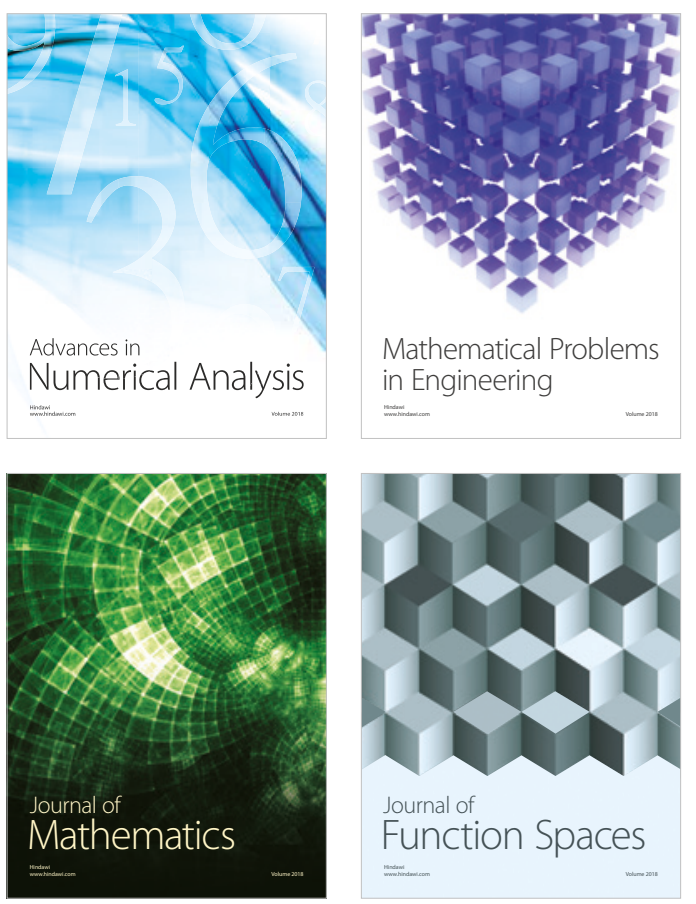

Mathematical Problems in Engineering

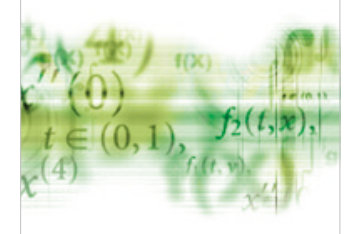

International Journal of

Differential Equations

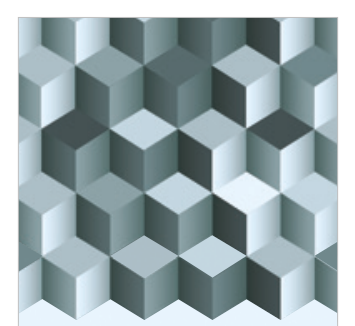

Journal of

Function Spaces

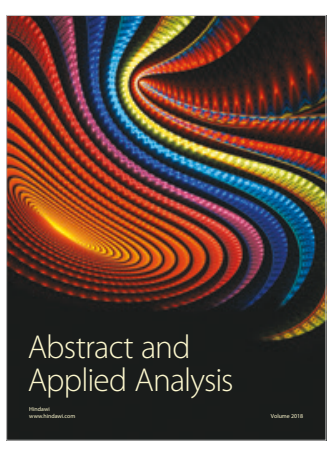

The Scientific

World Journal

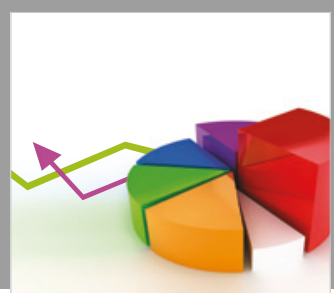

Journal of

Probability and Statistics
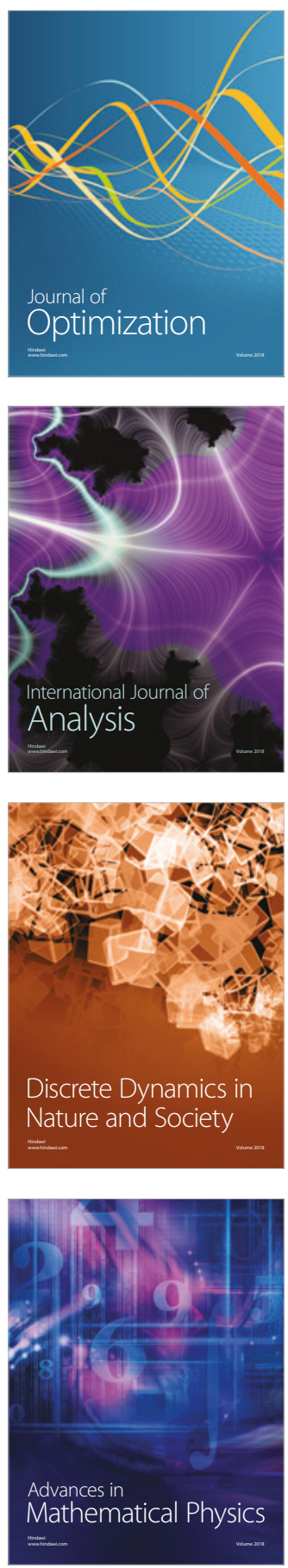